\title{
Molecular basis of broad neutralization against SARS-CoV-2 variants including Omicron by a human antibody
}

Bin $\mathrm{Ju}^{1,3, *, \#}$, Qingbing Zheng ${ }^{2,4, *}$, Huimin Guo ${ }^{1, *}$, Qing Fan ${ }^{1, *}$, Tingting $\mathrm{Li}^{2,4, *}$, Shuo Song ${ }^{1, *}$, Hui Sun ${ }^{2,4, *}$ Senlin Shen ${ }^{1}$, Xinrong Zhou ${ }^{1}$, Lin Cheng ${ }^{1}$, Wenhui $\mathrm{Xue}^{2,4}$, Lingyan Cui ${ }^{2,4}$, Bing Zhou ${ }^{1}$, Xiangyang Ge ${ }^{1}$, Haiyan Wang ${ }^{1}$, Miao Wang ${ }^{1}$, Shaowei $\mathrm{Li}^{2,4, \#}$, Ningshao Xia ${ }^{2,4, \#}$, Zheng Zhang ${ }^{1,3,5, \#}$ (1)

${ }^{1}$ Institute for Hepatology, National Clinical Research Center for Infectious Disease, Shenzhen Third People's Hospital; The Second Affiliated Hospital, School of Medicine, Southern University of Science and Technology, Shenzhen 518112, Guangdong Province, China.

${ }^{2}$ State Key Laboratory of Molecular Vaccinology and Molecular Diagnostics, School of Life Sciences, School of Public Health, Xiamen University, Xiamen 361102, Fujian Province, China.

${ }^{3}$ Guangdong Key laboratory for anti-infection Drug Quality Evaluation, Shenzhen 518112, Guangdong Province, China.

${ }^{4}$ National Institute of Diagnostics and Vaccine Development in Infectious Diseases, Xiamen University, Xiamen 361102, Fujian Province, China.

${ }^{5}$ Shenzhen Research Center for Communicable Disease Diagnosis and Treatment of Chinese Academy of Medical Science, Shenzhen 518112, Guangdong Province, China.

*These authors contributed equally to this work.

\#Correspondence:

zhangzheng1975@aliyun.com (Z.Z.)

nsxia@xmu.edu.cn (N.X.)

shaowei@xmu.edu.cn (S.L.)

jubin2013@163.com (B.J.)

One sentence summary: Structural and functional analyses reveal that a human antibody named VacW-209 confers broad neutralization against SARS-

CoV-2 variants including Omicron by recognizing a highly conserved epitope. 


\section{Abstract}

35 Omicron, a newly emerging SARS-CoV-2 variant, carried a large number of mutations in the spike protein leading to an unprecedented evasion from many neutralizing antibodies (nAbs). Here, we performed a head-to-head comparison of Omicron with other existing highly evasive variants in terms of their reduced sensitivities to antibodies, and found that Omicron variant is significantly more evasive than Beta and Mu variants. Of note, some key mutations occur in the conserved epitopes identified previously, especially in the binding sites of Class $4 \mathrm{nAbs}$, contributing to the increased Ab evasion. We also reported a broadly nAb (bnAb), VacW-209, which effectively neutralized all tested SARS-CoV-2 variants and even SARS-CoV. Finally, we determined six cryo-electron microscopy structures of VacW-209 complexed with the spike ectodomains of wild-type, Delta, Mu, C.1.2, Omicron, and SARS-CoV, and revealed the molecular basis of the broadly neutralizing activities of VacW-209 against SARS-CoV-2 variants. Overall, Omicron has once again raised the alarm over virus variation with significantly compromised neutralization. BnAbs targeting more conserved epitopes among variants will continue to play a key role in pandemic control and prevention. 


\section{Main text}

The coronavirus disease 2019 (COVID-19) caused by the infection of severe acute respiratory syndrome coronavirus 2 (SARS-CoV-2) is still a pandemic raging across the world. New variants, such as Alpha, Beta, Gamma, Delta, and Kappa, etc(1-4), keep emerging, causing a series of COVID-19 waves in different regions and countries worldwide. In addition to higher transmissibility and infectivity compared with the original wild-type (WT) virus, they could also escape, to a great extent, the neutralization of plasma and monoclonal neutralizing antibodies (nAbs) elicited by natural virus infection and vaccines(2, 3, 5, 6). For example, the Delta variant was first identified in India and has since become a long-time dominant variant rapidly spreading around the world due to its high transmissibility and some level of immune evasion by $n A b s(4,7)$. Its dominance can be revealed by the sequences collected on daily basis to the GISAID database since July 2021 , more than $90 \%$ of which were Delta lineage (Fig. 1A). The Beta has long been regarded as the variant with the greatest reduction in neutralizing capacity(8). However, a recent study showed that $\mathrm{Mu}$ variant, once driving the epidemic in Colombia, was more evasive than Beta in the neutralizing activity(9). The Mu variant shared similar mutations in receptor binding domain (RBD) of spike with Beta, R346K/E484K/N501Y and K417N/E484K/N501Y, respectively. After that, the C.1.2 variant was first identified and mainly reported in South Africa, also carrying three mutations (Y449H/E484K/N501Y) in RBD, which was similar to Beta(10). Although Beta, Mu, and C.1.2 variants did not give rise to a global pandemic like Delta (Fig. 1, $A$ and $B$ ), their occurrence were warning of possible variants with even more serious escape from the neutralization of pre-existing antibodies.

A new variant, Omicron, was first detected in South Africa in November 2021, and got dominant in many regions where Delta is prevalent with the short doubling time of cases(11). Omicron has been classified as a variant of concern (VOC) by the World Health Organization (WHO), whose spike protein carried more than 30 mutations including amino acid substitutions, deletions, and insertions(12). So many mutations, especially in the RBD (15 substitutions), 
85 had never appeared in any previous variants, suggesting that Omicron may 86 sharply escape the nAbs elicited by the WT virus or vaccine. Some recent studies indeed showed that Omicron significantly reduced the neutralization of plasma from convalescent patients or individuals immunized with several major SARS-CoV-2 vaccines. The Omicron variant also largely escaped the neutralization of monoclonal nAbs that have been approved for EmergencyUse-Administration (EUA), decreasing or abolishing their neutralizing activities(11-14). However, the molecular basis of the enhanced antibody evasion is not well defined, especially for comparison of the Omicron to Beta, Delta, Mu, and C.1.2 variants with more serious escape from the nAbs. In addition, it lacks a head-to-head comparison of the structural and functional characterization for the novel broadly nAb (bnAb) against these variants and even SARS-CoV.

We previously developed and validated a pseudovirus neutralization assay to evaluate the neutralizing activities of plasma and mAbs against the WT SARSCoV-2 and variants(15-17). On top of it, we constructed Mu, C.1.2, and Omicron pseudoviruses (Fig. 1C). To make a comprehensive evaluation of the susceptibility of Omicron, we summarized plasma samples from 19 individuals who were infected with the WT virus and had recovered from COVID-19 in the first wave of pandemic, and then measured and compared their neutralizing activities against the WT, Beta, Delta, Mu, C.1.2, and Omicron variants, head to head (Fig. 1D and fig. S1). In consistence with a previous study(9), the $\mathrm{Mu}$ variant showed more serious resistance to nAbs than Beta (15.4-fold vs. 9.6fold). In contrast, the Delta and C.1.2 variants showed the relatively weaker escape from the neutralization of pre-existing antibodies (3.2-fold and 2.3-fold,

111 respectively). Remarkably, we found that the geometric mean titer (GMT) of 112 nAbs against Omicron decreased, three folds greater than Mu (55 vs. 163), and 113 over 40 folds compared with that against WT $(G T M=2508)$. More seriously, 114 some plasma (3/19) lost their neutralizing activities against Omicron, whose 50\% 115 inhibitory dilution $\left(I_{50}\right)$ was less than the dilution of 1:20 (Fig. 1E). Overall, the 116 largely enhanced antibody evasion by Omicron variant is unprecedented, and 117 the appearance of Omicron is another warn of virus escape. 
119 To study the mechanism of antibody escape, we summarized and prepared a 120 panel of 12 published nAbs binding to RBD of SARS-CoV-2 with clear structural 121 information. The RBD recognizes the cellular receptor (angiotensin-converting 122 enzyme 2, ACE2) and mediates the virus entry into the host cell, which is the 123 primary target for blocking viral infection $(18,19)$. Therefore, RBD-specific nAbs 124 contribute a lot to the neutralization of SARS-CoV-2. Here, we used these 12 $125 \mathrm{nAbs}$ recognizing diverse epitopes to mimic the polyclonal antibodies in plasma 126 to explore what kind of nAbs were mostly affected by the mutations of variants 127 and the molecular basis of antibody evasion. In general, the nAbs can be 128 classified into classes 1, 2, 3, and 4 based on the competition with ACE2 and 129 the binding model to RBD (fig. S4A)(20, 21). The Beta mainly escaped from 130 the neutralization of nAbs of Class 1 and 2, yet Delta, Mu, and C.1.2 partially 131 or totally escaped the nAbs of Class 2 and 3 we tested. Notely, the neutralizing 132 and binding activities of Class 4 antibodies were not affected by these variants 133 (Fig. 1, F and G; fig. S2 and S3). However, Omicron abolished the 134 neutralization of nearly all nAbs we tested (11/12, except S309) across Class 1 135 to 4 (Fig. 1F), explaining why Omicron has the most serious antibody evasion 136 so far.

138 The structural analysis further showed that some key mutations were located 139 in or near the footprint of nAbs on the RBD (fig. S4A). The K417N and Q493R 140 substitutions mainly affect the recognition of Class $1 \mathrm{nAbs}$ to Omicron RBD. For 141 example, the overall binding mode of CB6 had been changed. CB6 interacts to 142 the K417 residue on RBD with Y33, Y52, and D104 residues of heavy chain 143 through hydrogen bond and salt bridge interactions. Q493 is another key 144 epitope residue for Class $1 \mathrm{nAbs}$ binding to RBD, which make a salt bridge with 145 heavy-chain Y109 residue (fig. S4B). E484 on WT RBD forms hydrogen bond 146 and salt bridge interactions with R100, R102, and D106 of BD-368-2 heavy 147 chain. Therefore, mutations at E484 usually result in complete insensitivity of 148 Class 2 nAbs (fig. S4C). The mechanisms of Class 1 and 2 nAbs escape 149 mentioned above have been stated on Beta, Gamma, and Kappa variants in 150 previous reports(2, 5, 22-24). For REGN10987, an antibody of Class 3, the 
151 G446S mutation may diminish the binding of this class of nAbs to RBD (fig.

152 S4D). As mentioned above, the Omicron was the first variant to escape Class

1534 nAbs. The structural analysis of H014 showed that S371L may mediate the 154 resistance of Omicron to this class of nAbs (fig. S4E). Previously, REGN10987155 like nAbs and Class 4 nAbs were considered to have the most broadly 156 neutralizing activities, whose targeting epitopes were relatively conserved 157 among various SARS-CoV-2 variants(4, 5, 7, 25, 26). G446S and S371L are 158 both newly identified signature mutations of the Omicron, which confer the great 159 antibody resistance and have changed our understanding about the bnAbs. 160 Fortunately, some minority of existing nAbs are effective to Omicron. Consistent 161 with previous studies $(11,27)$, S309 retains a strong binding affinity to Omicron162 RBD and an effective neutralizing activity (Fig. 1, F and G), despite the 163 existence of G339 and N440 residues as part of its epitopes (fig. S4D).

Then we explore other bnAbs against Omicron, especially those bind to distinct epitopes away from S309. Previously, we identified 9 monoclonal nAbs from 2 individuals immunized with the SARS-CoV-2 inactivated vaccine and measured their cross-neutralizing activities against Kappa and Delta(17). Here, we further evaluated the neutralization of these $n A b s$ against other important variants including Alpha, Beta, Gamma, Mu, especially Omicron, and so on (Fig. 2A and fig. S5). Their neutralizing breadths ranged from $54 \%$ to $100 \%$ in the tested 13 SARS-CoV-2 pseudoviruses, and a few of the nAbs (3/9) could still neutralize Omicron. VacW-92 and VacW-120 binding to overlapped epitopes with $S 309$ (17) effectively neutralized Omicron $(1.246 \mu \mathrm{g} / \mathrm{mL}$ and $0.273 \mu \mathrm{g} / \mathrm{mL}$, respectively) but not Delta. VacW-209 could neutralize all variants tested here with a high potency (Geometric $\mathrm{IC}_{50}=0.063 \mu \mathrm{g} / \mathrm{mL}$ ). Meanwhile, the binding affinities of VacW-209 to Mu, C.1.2, and Omicron RBD proteins may contribute 178 to its broadly neutralizing activity (Fig. 2B and fig. S6). SARS-CoV is closely 179 related to SARS-CoV-2 sharing $80 \%$ of amino acid sequence identity in their 180 spike proteins(28). Therefore, we also detected the cross-reaction of VacW-209 181 to SARS-CoV, which displayed both highly neutralizing activity $(0.141 \mu \mathrm{g} / \mathrm{mL})$ 182 and binding affinity (0.540 nM) (Fig. 2, C and D). VacW-209 strongly competed 183 with ACE2 for binding to RBD, revealing its neutralizing mechanism of high 
184 potency (Fig. 2E). Then, we measured the competition of VacW-209 with 15 185 typical nAbs of Class 1 to 4, which revealed that VacW-209 bound to an epitope 186 overlapped with that of both Class 1 and Class 4 nAbs (Fig. 2F). It was found 187 that VacW-209 did not compete with two approved nAb drugs (REGN10987 188 and S309), suggesting that it could be used both independently and in 189 combination with these nAbs. We therefore evaluated the neutralizing activities 190 of VacW-209+REGN10987 and VacW-209+S309 against WT, Beta, Delta, and 191 Omicron,respectively (Fig. 2, G and H). REGN10987 completely lost its 192 neutralization against Omicron, but it could be effectively rescued through the 193 combination with VacW-209. Considering that S309 and VacW-209 are both 194 potent nAbs against all identified SARS-CoV-2 variants so far and SARS-CoV, 195 the combination will open up the way against virus escape in the future.

197 To define the structural basis of the broadly neutralizing activity of VacW-209, 198 we resolved the cryo-electron microscopy (cryo-EM) structures of the antigen199 binding fragment (Fab) of VacW-209 in complex with the spike proteins of 200 SARS-CoV-2 WT, Delta, Mu, C.1.2, Omicron, and SARS-CoV, respectively. Six 201 cryo-EM structures of trimeric spike based immune complexes at 2.98-3.45 $\AA$ 202 revealed nearly identical binding modes of VacW-209 (Fig. 3, A to F; fig. S7 to 203 S12; and table S1). Three VacW-209 Fabs bound to a completely opened S 204 with three "up" RBDs. We then performed the focus refinement of regions of 205 Fab-bound RBDs of WT, Delta, Mu, C.1.2, and Omicron, while the Fab-bound 206 SARS-CoV RBD was weakly resolved due to structural flexibility (Fig. 3, G to 207 K). High-resolution structures revealed that the binding epitope of VacW-209 208 completely evaded the key RBD mutations in variants of Delta, Mu, C.1.2, and 209 rarely overlapped with mutations in Omicron (Fig. 3, H to K, lower). The 210 footprints of VacW-209 on WT-RBD and Omicron-RBD were slightly different, 211 and three mutations in Omicron (K417N, S373P, and S375F) were involved in 212 the nAb-RBD interaction (Fig. 3, L and M).

214 We next analyzed the interaction details of VacW-209 binding to WT and 215 Omicron spikes, respectively, and revealed that VacW-209 mainly used its 216 extremely long heavy loop at complementarity determining region (CDR) 3 to 
217 mediate spike protein recognition. Besides, the light chain CDR2 and D34 from 218 LCDR1 were also involved in nAb-RBD interactions (Fig. 3, N to Q). For WT219 RBD, residues $371,379,408,414$, and 415 formed a total of 11 hydrogen bonds 220 and 2 salt bridges to VacW-209 and created an interaction network between 221 them (Fig. 3, N and O). The heavy chain R106 inserted its long side chain into 222 the pocket formed by RBD aa. 371-385, which contained three key mutations 223 of Omicron (S371L/S373P/S375F) (fig. S13, A to C). Although VacW-209 224 showed a decreased neutralization against Omicron compared with that 225 against WT, to some extent (Fig. 2A), our structural analysis showed that the 226 mutations surrounding RBD aa. 371-385 loop seemed not to obviously affect 227 the binding of VacW-209 since the S373P and S375F built three new hydrogen 228 bonds with heavy chain R106 (Fig. 3P). Other Omicron mutations were not 229 involved in the hydrogen bond interactions, and there were a total of 12 230 hydrogen bonds and 1 salt bridges formed (Fig. 3, P and Q), which were 231 comparable in total to that in WT. We further found that the binding of VacW232209 to Omicron RBD need a slight conformational change of 371-385 loop 233 containing S371L/S373P/S375F mutations (fig. S13, D and E), which may 234 partly account for the reduced neutralization of VacW-209 against Omicron 235 variant.

237 Finally, we compared the binding mode of VacW-209 to several nAbs of Class 2381 to 4 and defined a new binding mode of VacW-209 which bound to an epitope 239 between Class 1 and Class 4 , yet not overlapping with that of Class 2 or Class 2403 (Fig. 4A). Despite some minor differences in details, the binding of VacW-209 241 to RBDs of SARS-CoV-2 WT, Delta, Mu, C.1.2, and Omicron were all mediated 242 by the long HCDR3 (in particular R106, Y116, and D119), LCDR2 (in particular 243 Y51, N55, and S58), and LCDR1 residue D34 (Fig. 4, B to F). We also explored 244 the potential binding sites of VacW-209 on other variant RBDs as well as SARS245 CoV RBD based on the binding characterization revealed in the WT-S2P:VacW246209 (Fig. 4, G to K). The epitope of VacW-209 nearly excluded all of above 247 RBD mutations and was highly conserved between SARS-CoV-2 and SARS248 CoV (Fig. 4, C to K). The epitope was mainly comprised of RBD aa. 376-385 249 and 405-416, which was highly conserved among SARS-CoV-2 variants and 
250 there were only three amino acid substitutions between SARS-CoV-2 and 251 SARS-CoV (Fig. 4L).

253 The similar binding mode of VacW-209 was also found in some previously 254 reported nAbs including C118, C022, S2X35, and S2X259(29-31) (Fig. 4M). 255 Available structural information revealed that the aforementioned four nAbs and 256 VacW-209 shared lots of epitope residues located in conserved RBD aa. 376257385 and 405-416, while with diverse coverage of key mutations of Omicron (Fig. 258 4N; fig. S14, A to E). Of these mutations, S371L, S373P, S375F, K417N, 259 N501Y, and Y505H were structurally close to or involved in the binding epitopes 260 of VacW-209-like nAbs. In the head-to-head comparison, although C022 and 261 S2X35 showed significant reductions of neutralization against Omicron, these 262 VacW-209-like nAbs generally maintained effectively neutralizing and binding 263 activities to various SARS-CoV-2 variants and even SARS-CoV (fig. S14, F 264 and G; fig. S15). The molecular mechanism underlying why these similar nAbs 265 display diverse neutralizing activities need to be elucidated in the future.

In conclusion, the bnAb described here, VacW-209, idenfies a highly conserved epitope on the RBDs among SARS-CoV-2 variants overlapping with the ACE2binding site, responsible for its potent neutralization. VacW-209 could strongly compete with Class 4 nAbs, indicating the potential cross-neutralization against sarbecoviruses. These VacW-209-like nAbs shared a similar antibody response to both SARS-CoV-2 and SARS-CoV, highlighting a candidate target for the

273 universal vaccine design. As a promising antibody therapeutics, VacW-209, 274 alone or in combination with $\$ 309$, could also be used as countmeasure against SARS-CoV-2 variants including Omicron and even other forthcoming sarbecoviruses in the future.

\section{References}

279 1. A. Muik et al., Neutralization of SARS-CoV-2 lineage B.1.1.7 pseudovirus by BNT162b2 vaccine-elicited human sera. Science, (2021).

281 2. R. Wang et al., Analysis of SARS-CoV-2 variant mutations reveals 282 neutralization escape mechanisms and the ability to use ACE2 receptors 
from additional species. Immunity, (2021).

284 3. P. Wang et al., Increased resistance of SARS-CoV-2 variant P.1 to antibody neutralization. Cell Host Microbe 29, 747-751 e744 (2021).

4. C. Liu et al., Reduced neutralization of SARS-CoV-2 B.1.617 by vaccine and convalescent serum. Cell, (2021).

5. P. Wang et al., Antibody Resistance of SARS-CoV-2 Variants B.1.351 and B.1.1.7. Nature, (2021).

6. M. McCallum et al., SARS-CoV-2 immune evasion by the B.1.427/B.1.429 variant of concern. Science, (2021).

7. D. Planas et al., Reduced sensitivity of SARS-CoV-2 variant Delta to antibody neutralization. Nature, (2021).

8. C. Lucas et al., Impact of circulating SARS-CoV-2 variants on mRNA vaccine-induced immunity. Nature, (2021).

9. K. Uriu et al., Neutralization of the SARS-CoV-2 Mu Variant by Convalescent and Vaccine Serum. N Engl J Med 385, 2397-2399 (2021).

10. T. Tada et al., Neutralization of Mu and C.1.2 SARS-CoV-2 Variants by Vaccine-elicited Antibodies in Individuals With and Without Previous History of Infection. bioRxiv, (2021).

11. L. Liu et al., Striking antibody evasion manifested by the Omicron variant of SARS-CoV-2. Nature, (2021).

12. D. Planas et al., Considerable escape of SARS-CoV-2 Omicron to antibody neutralization. Nature, (2021).

13. E. Cameroni et al., Broadly neutralizing antibodies overcome SARS-CoV-2 Omicron antigenic shift. Nature, (2021).

14. S. Cele et al., Omicron extensively but incompletely escapes Pfizer BNT162b2 neutralization. Nature, (2021).

15. Q. Zhang et al., Potent and protective IGHV3-53/3-66 public antibodies and their shared escape mutant on the spike of SARS-CoV-2. Nat Commun 12, 4210 (2021).

16. L. Cheng et al., Impact of the N501Y substitution of SARS-CoV-2 Spike on neutralizing monoclonal antibodies targeting diverse epitopes. Virol J 18, 87 (2021).

17. L. Cheng et al., Cross-neutralization of SARS-CoV-2 Kappa and Delta 
variants by inactivated vaccine-elicited serum and monoclonal antibodies. Cell Discov 7, 112 (2021).

18. L. Liu et al., Potent neutralizing antibodies directed to multiple epitopes on SARS-CoV-2 spike. Nature, (2020).

19. B. Ju et al., Human neutralizing antibodies elicited by SARS-CoV-2 infection. Nature, (2020).

20. C. O. Barnes et al., SARS-CoV-2 neutralizing antibody structures inform therapeutic strategies. Nature, (2020).

21. R. Yan et al., Structural basis for bivalent binding and inhibition of SARSCoV-2 infection by human potent neutralizing antibodies. Cell Res $\mathbf{3 1}$, 517-525 (2021).

22. S. M. Gobeil et al., Effect of natural mutations of SARS-CoV-2 on spike structure, conformation, and antigenicity. Science, (2021).

23. M. Yuan et al., Structural and functional ramifications of antigenic drift in recent SARS-CoV-2 variants. Science, (2021).

24. M. McCallum et al., Molecular basis of immune evasion by the Delta and Kappa SARS-CoV-2 variants. Science 374, 1621-1626 (2021).

25. D. Zhou et al., Structural basis for the neutralization of SARS-CoV-2 by an antibody from a convalescent patient. Nat Struct Mol Biol, (2020).

26. Z. Lv et al., Structural basis for neutralization of SARS-CoV-2 and SARSCoV by a potent therapeutic antibody. Science, (2020).

27. Y. Cao et al., Omicron escapes the majority of existing SARS-CoV-2 neutralizing antibodies. Nature, (2021).

28. D. Pinto et al., Cross-neutralization of SARS-CoV-2 by a human monoclonal SARS-CoV antibody. Nature, (2020).

29. T. N. Starr et al., SARS-CoV-2 RBD antibodies that maximize breadth and resistance to escape. Nature 597, 97-102 (2021).

30. M. A. Tortorici et al., Broad sarbecovirus neutralization by a human monoclonal antibody. Nature 597, 103-108 (2021).

31. C. A. Jette et al., Broad cross-reactivity across sarbecoviruses exhibited by a subset of COVID-19 donor-derived neutralizing antibodies. Cell Rep 36, 109760 (2021). 


\section{Acknowledgments}

350 We thank all participants who recovered from the COVID-19 and who received SARS-CoV-2 inactivated vaccines. We also thank all of the healthy providers from Shenzhen Third People's Hospital for the work they have done. This study was supported by the National Science Fund for Distinguished Young Scholars (82025022), the National Natural Science Foundation of China (92169204, 82002140, 82171752, 82101861, 81991491), the National Key Plan for Scientific Research and Development of China (2021YFC0864500), the Guangdong Basic and 2020A1515110656), the Shenzhen Science and Technology Program (RCYX20200714114700046), and the Science and Technology Innovation JSGG20200807171401008, JCYJ20190809115617365, SGG20210901145200002).

\section{Author contributions}

Z.Z. is the principal investigator of this study. Z.Z., N.X., S.L., and B.J. H.S. performed all experiments and analyzed data together with S. Shen, X.Z., L.Cheng, W.X., L.Cui, B.Z., X.G., H.W., and M.W.. Z.Z., N.X., S.L., B.J., and Q.Z. participated in discussion of the results and wrote the manuscript. All authors read and approved this version of manuscript.

\section{Data and materials availability}

374 We are happy to share reagents and information in this study upon request.

375 Structure coordinates are deposited in the Protein Data Bank under accession 376 codes XXXX (WT-S2P:VacW-209), XXXX (Delta-S6P:VacW-209) XXXX (Mu377 S6P:VacW-209), XXXX (C.1.2-S6P:VacW-209), and XXXX (Omicron378 S6P:VacW-209). The corresponding EM density maps have been deposited in 379 the Protein Data Bank under accession numbers XXXX (WT-S2P:VacW-209), XXXX (Delta-S6P:VacW-209) XXXX (Mu-S6P:VacW-209), XXXX (C.1.2- 
381 S6P:VacW-209), XXXX (Omicron-S6P:VacW-209), and XXXX (SARS-CoV382 S6P:VacW-209).

383

384 Conflict of interest

385 The authors declare that they have no conflict of interest. 
A

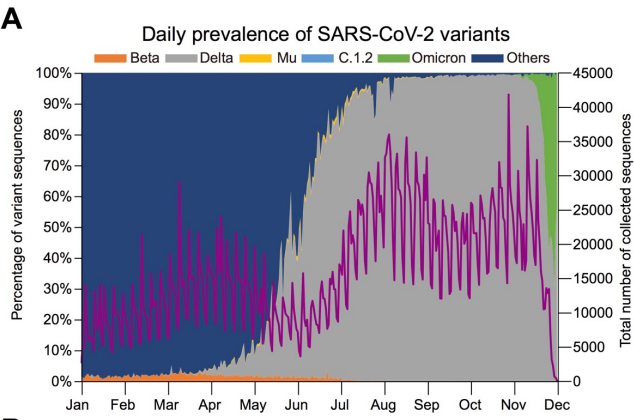

B

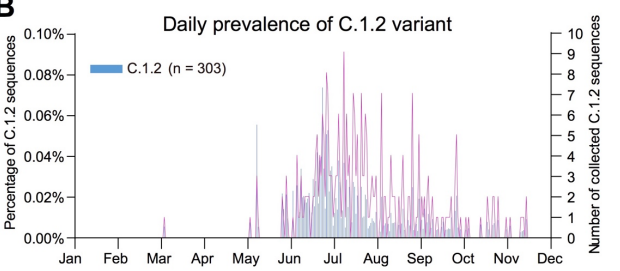

D

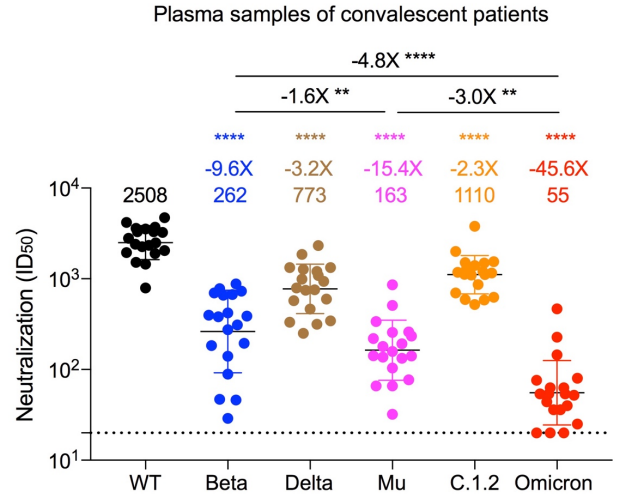

F

\begin{tabular}{|c|c|c|c|c|c|c|c|}
\hline Class & mAbs (IC50) & WT & Beta & Delta & $\mathrm{Mu}$ & C.1.2 & Omicron \\
\hline \multirow{3}{*}{ Class 1} & CB6 & 0.012 & $>50$ & 0.149 & 0.150 & 0.230 & $>50$ \\
\hline & REGN10933 & 0.002 & 1.492 & 0.002 & 0.081 & 0.025 & $>50$ \\
\hline & CC12.1 & 0.009 & $>50$ & 0.612 & 0.101 & 0.053 & $>50$ \\
\hline \multirow{3}{*}{ Class 2} & BD-368-2 & 0.001 & $>50$ & 5.079 & $>50$ & $>50$ & $>50$ \\
\hline & P2B-2F6 & 0.029 & $>50$ & $>50$ & $>50$ & $>50$ & $>50$ \\
\hline & C144 & 0.001 & $>50$ & 0.005 & $>50$ & $>50$ & $>50$ \\
\hline \multirow{3}{*}{ Class 3} & REGN10987 & 0.002 & 0.002 & 0.003 & 0.001 & 0.002 & $>50$ \\
\hline & C110 & 0.005 & 7.235 & $>50$ & 6.787 & $>50$ & $>50$ \\
\hline & s309 & 0.093 & 0.042 & 0.042 & 0.070 & 0.219 & 0.158 \\
\hline \multirow{3}{*}{ Class 4} & HO14 & 0.112 & 0.689 & 0.385 & 0.556 & 0.098 & $>50$ \\
\hline & EY6A & 3.806 & 1.592 & 4.997 & 6.874 & 3.658 & $>50$ \\
\hline & S304 & 2.687 & 3.394 & 4.484 & 8.549 & 4.984 & $>50$ \\
\hline
\end{tabular}

C
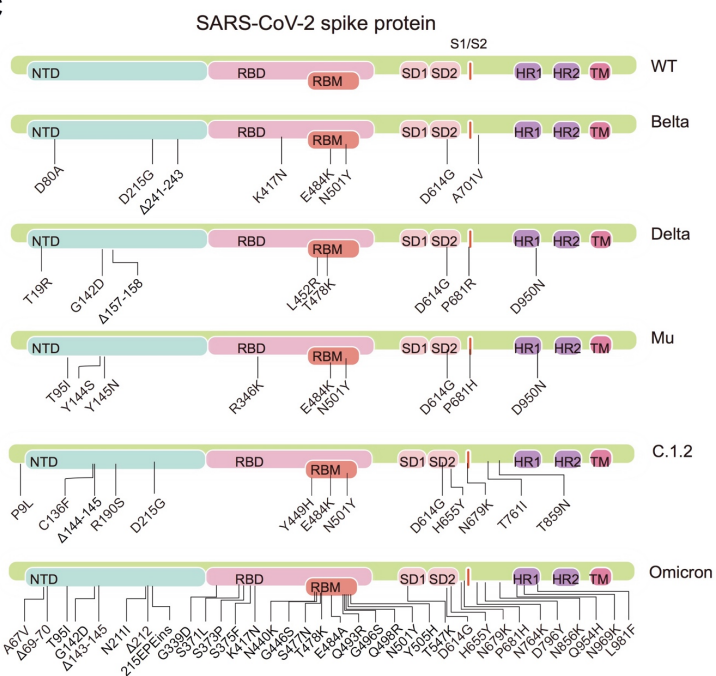

E

\begin{tabular}{|c|c|c|c|c|c|c|c|}
\hline Patients & WT & Beta & Delta & $\mathrm{Mu}$ & $\begin{array}{ll}\text { C.1.2 } \\
\end{array}$ & Omicron & \\
\hline Donor-1 & 4721 & 672 & 1206 & 860 & 3784 & 227 & \\
\hline Donor-2 & 2341 & 382 & 746 & 192 & 1121 & 54 & \\
\hline Donor-3 & 3241 & 421 & 1270 & 179 & 1542 & 80 & \\
\hline Donor-4 & 1905 & 194 & 756 & 141 & 1299 & 54 & \\
\hline Donor-5 & 2486 & 779 & 1333 & 259 & 1491 & 467 & \\
\hline Donor-6 & 3599 & 658 & 938 & 507 & 1509 & 54 & \\
\hline Donor-7 & 2406 & 29 & 251 & 77 & 583 & 25 & \\
\hline Donor-8 & 2048 & 140 & 794 & 133 & 592 & 63 & \\
\hline Donor-9 & 791 & 47 & 575 & 32 & 520 & 20 & \\
\hline Donor-10 & 3540 & 310 & 989 & 256 & 1996 & 145 & $\begin{array}{l}(\mathbb{D} 50) \\
\text { (a) }\end{array}$ \\
\hline Donor-11 & 1522 & 46 & 344 & 66 & 1197 & 20 & 5000 \\
\hline Donor-12 & 1451 & 184 & 315 & 158 & 626 & 40 & 4000 \\
\hline Donor-13 & 1947 & 89 & 333 & 66 & 698 & 20 & 3000 \\
\hline Donor-14 & 3295 & 389 & 464 & 137 & 1102 & 44 & 2000 \\
\hline Donor-15 & 4184 & 699 & 2319 & 337 & 1404 & 36 & 1000 \\
\hline Donor-16 & 2796 & 878 & 1327 & 220 & 1156 & 76 & 500 \\
\hline Donor-17 & 3294 & 274 & 596 & 104 & 1114 & 36 & 200 \\
\hline Donor-18 & 3704 & 397 & 1856 & 234 & 1175 & 63 & 21 \\
\hline Donor-19 & 2264 & 731 & 1085 & 142 & 864 & 52 & 20 \\
\hline
\end{tabular}

G

\begin{tabular}{|c|c|c|c|c|c|c|}
\hline Class & mAbs (Ko) & WT & $\mathrm{Mu}$ & $\begin{array}{ll}\text { C.1.2 } \\
\end{array}$ & Omicron & \multirow{7}{*}{$\begin{array}{l}\text { Binding affinity } \\
\left(\begin{array}{l}\mathrm{Ko}, \mathrm{nM})\end{array}\right.\end{array}$} \\
\hline \multirow{3}{*}{ Class 1} & \multirow{3}{*}{$\begin{array}{l}\text { CB6 } \\
\text { REGN10933 } \\
\text { CC12.1 }\end{array}$} & 0.349 & 3.47 & 5.58 & n.b. & \\
\hline & & 0.0585 & 1.23 & 1.47 & n.b. & \\
\hline & & 0.680 & 2.93 & 2.17 & n.b. & \\
\hline \multirow{3}{*}{ Class 2} & $\mathrm{BD}-368-2$ & 0.0327 & n.b. & n.b. & n.b. & \\
\hline & P2B-2F6 & 0.956 & n.b. & n.b. & n.b. & \\
\hline & C144 & 0.563 & n.b. & n.b. & n.b. & \\
\hline \multirow{3}{*}{ Class 3} & REGN10987 & 0.365 & 0.527 & 0.375 & n.b. & 0.01 \\
\hline & C110 & 0.156 & 24.4 & n.b. & n.b. & 0.1 \\
\hline & s309 & $<0.0200$ & $<0.0300$ & $<0.0200$ & 2.13 & 1 \\
\hline \multirow{3}{*}{ Class 4} & H014 & 0.126 & 0.117 & 0.114 & n.b. & 10 \\
\hline & EY6A & 0.270 & 0.298 & 0.240 & 0.997 & ${ }^{20}$ \\
\hline & S304 & 0.0941 & 0.0772 & 0.0365 & 0.210 & n.b. \\
\hline
\end{tabular}

Figure 1. Resistance of SARS-CoV-2 variants including Omicron to the neutralization by convalescent plasma and monoclonal nAbs.

390 (A) Daily prevalence of the variants and their distributions in all collected SARS391 CoV-2 sequences in the world deposited to GISAID in 2021. The left y-axis 392 indicates the percentage of each variant collected everyday, which is displayed 393 by areas in different colors. Orange: Beta, Gray: Delta, Yellow: Mu, Light blue: 394 C.1.2, Green: Omicron, Dark blue: Others. The right y-axis indicates the total 395 number of collected sequences each day, which is displayed by the purple line.

396 (B) The prevalence of C.1.2 variant was separated to shown due to its rarity 
397 among the reported sequences. (C) The landscape of key mutations in spike 398 proteins of SARS-CoV-2 variants used in this study. The full-length mutated 399 spike genes were synthesized to construct pseudoviruses. The WT, Beta, Delta, $400 \mathrm{Mu}, \mathrm{C} .1 .2$, and Omicron variants were shown from top to bottom. (D) The 401 neutralization of WT SARS-CoV-2 and variants by plasma samples of 19 402 convalescent patients infected with the WT virus and recovered from the first 403 wave of COVID-19 pandemic. The GMT of nAbs, fold change, and statistical 404 analysis were calculated and noted on the top of each column. The data are 405 shown in Geometric mean \pm SD. "-" indicates decreased neutralization activity. 406 Fold changes in GMTs were compared between each variant and WT or 407 between two variants. Statistical analysis was performed with a paired $t$ test 408 using GraphPad Prism 9 software. ${ }^{* *}: \mathrm{P}<0.01,{ }^{* * *}: \mathrm{P}<0.0001$. The horizontal 409 dashed line indicates the limit of detection (1:20 dilution) for the neutralizing 410 assay. The non-neutralizing data below the limit were set to 20 for visualization. 411 (E) The neutralization of each plasma sample against WT and variants was 412 represented in $I D_{50}$ value. $(F)$ The neutralization $\left(I_{50}\right)$ of 12 representative 413 nAbs of Class 1 to 4 against WT SARS-CoV-2 and variants. The cutoff value of 414 neutralization was set as $50 \mu \mathrm{g} / \mathrm{mL}$. (G) The binding affinity $\left(K_{D}\right)$ of 12 415 representative nAbs to RBD proteins of WT SARS-CoV-2 and variants by SPR. 416 The neutralizing potency or binding affinity is highlighted in different colors. Red: 417 high, Yellow: moderate, Green: weak, Gray: non-neutralizing or not binding 418 (n.b.). The data are means of two independent experiments. 
A

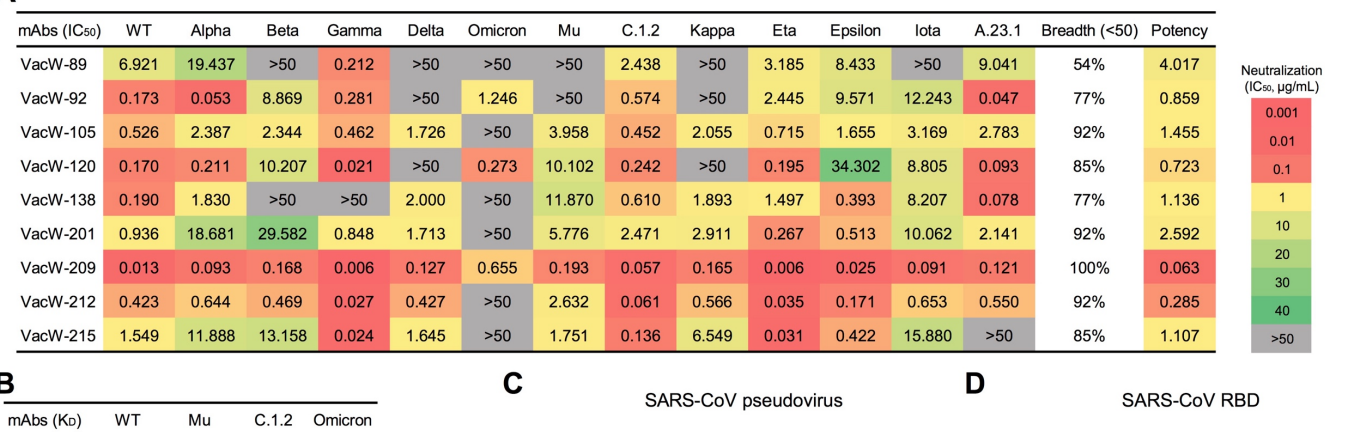

\begin{tabular}{lcccc}
\hline mAbs (Ko) & WT & Mu & C.1.2 & Omicron \\
\hline VacW-89 & 3.29 & 5.12 & 4.47 & 3.55
\end{tabular}

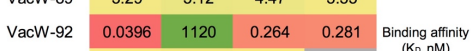

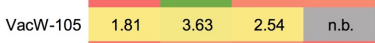

$\begin{array}{lllll}\text { VacW-120 } & 0.117 & 0.408 & 0.347 & 0.0905 \\ \text { Vach } & 1.30 & 21.2 & 125 & 0.6\end{array}$

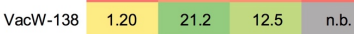

$\begin{array}{lllll}\text { VacW-201 } & 1.68 & 3.60 & 4.10 & \text { n.b. }\end{array}$

$\begin{array}{lllll}\text { VacW-209 } & 0.0268 & 0.0200 & 0.0229 & 0.722\end{array}$

\begin{tabular}{|l|l|l|l|l|l}
\hline VacW-212 & 0.560 & 0.700 & 0.808 & n.b.
\end{tabular}

\begin{tabular}{l|ccc|c|c|} 
VacW-215 & 0.580 & 1.89 & 1.12 & n.b. \\
\hline
\end{tabular}

E Compettion with ACE2 $\quad \mathbf{F}$
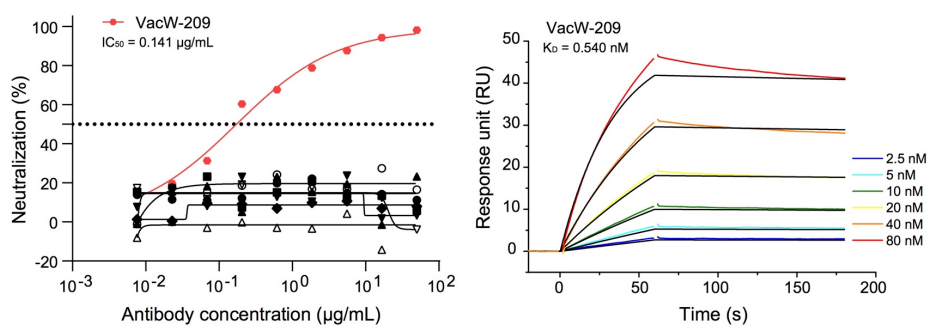

G

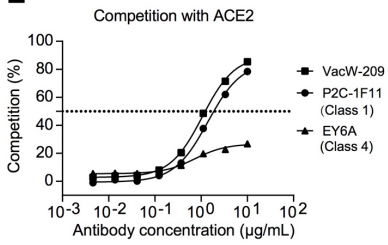

Competition with VacW-209
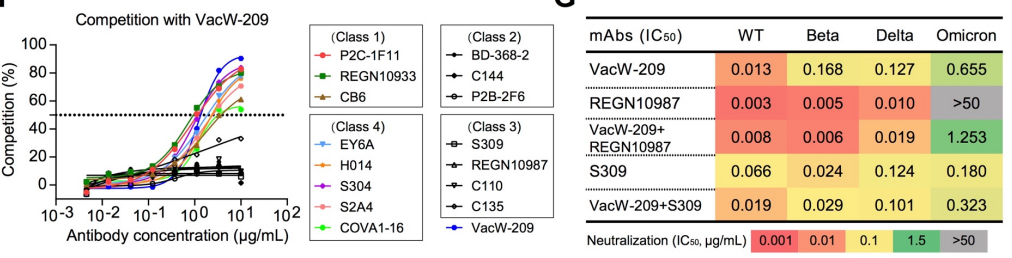

H

wT
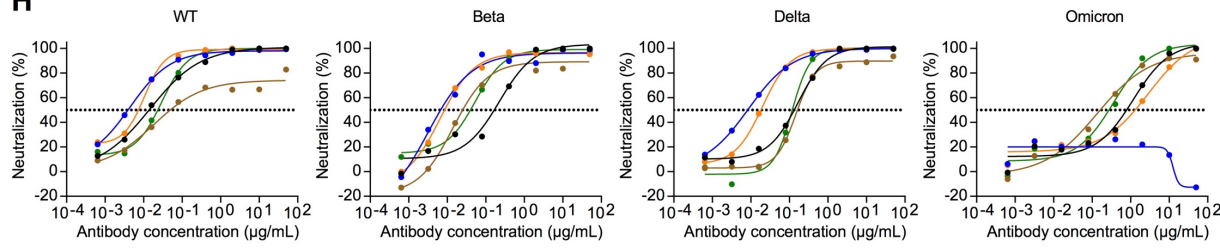

$\rightarrow$ VacW-209
$\rightarrow$ REGN10987

- VacW-209+REGN10987

$\rightarrow 5309$

VacW-209+\$309

420 Figure 2. Identification of bnAbs against SARS-CoV-2 variants including

421 Omicron.

422 (A) The neutralization $\left(\mathrm{IC}_{50}\right)$ of $9 \mathrm{nAbs}$ isolated from individuals vaccinated with

423 WT SARS-CoV-2 vaccine against 13 tested pseudoviruses. The cutoff value of 424 neutralization was set as $50 \mu \mathrm{g} / \mathrm{mL}$. The neutralizing breadth was calculated as 425 the percentage of viruses neutralized by each nAb. Geometric mean potency

426 was calculated by the neutralizing value of less than $50 \mu \mathrm{g} / \mathrm{mL}$. (B) The binding 427 affinity $\left(K_{D}\right)$ of 9 nAbs to RBD proteins of WT SARS-CoV-2 and variants by SPR. 428 The neutralizing and/or binding activities of 9 nAbs to WT, Delta, and Kappa 429 have been reported in our previous study(17), which are re-tested here for a 430 head-to-head comparison with other variants. (C) The neutralizing activities of 4319 nAbs against SARS-CoV. VacW-209 is marked in red, the other non432 neutralizing mAbs are marked in black. (D) The binding affinity of VacW-209 to 
433 SARS-CoV RBD by SPR. (E) Competition ELISA of VacW-209 with human 434 ACE2 for binding to WT RBD. P2C-1F11 was a known competitive antibody as 435 a positive control. EY6A was a known non-competitive antibody as a negative 436 control. (F) Competition ELISA of VacW-209 with 15 representatives nAbs of 437 four classes and with itself for binding to WT RBD. The neutralizing activities of 438 VacW-209 combined with REGN10987 or S309 against WT, Beta, Delta, and 439 Omicron were represented in $I_{50}$ values $(\mathbf{G})$ and curves $(\mathbf{H})$. The data 440 represented here are means of at least two independent experiments. The 441 neutralizing potency or binding affinity is highlighted in different colors. Red: 442 high, Yellow: moderate, Green: weak, Gray: non-neutralizing or not binding 443 (n.b.). The curve of neutralization, binding affinity, or competition was one out 444 of similar results. A cutoff value of $50 \%$ is indicated by a horizontal dashed line 445 in neutralization and competition. 


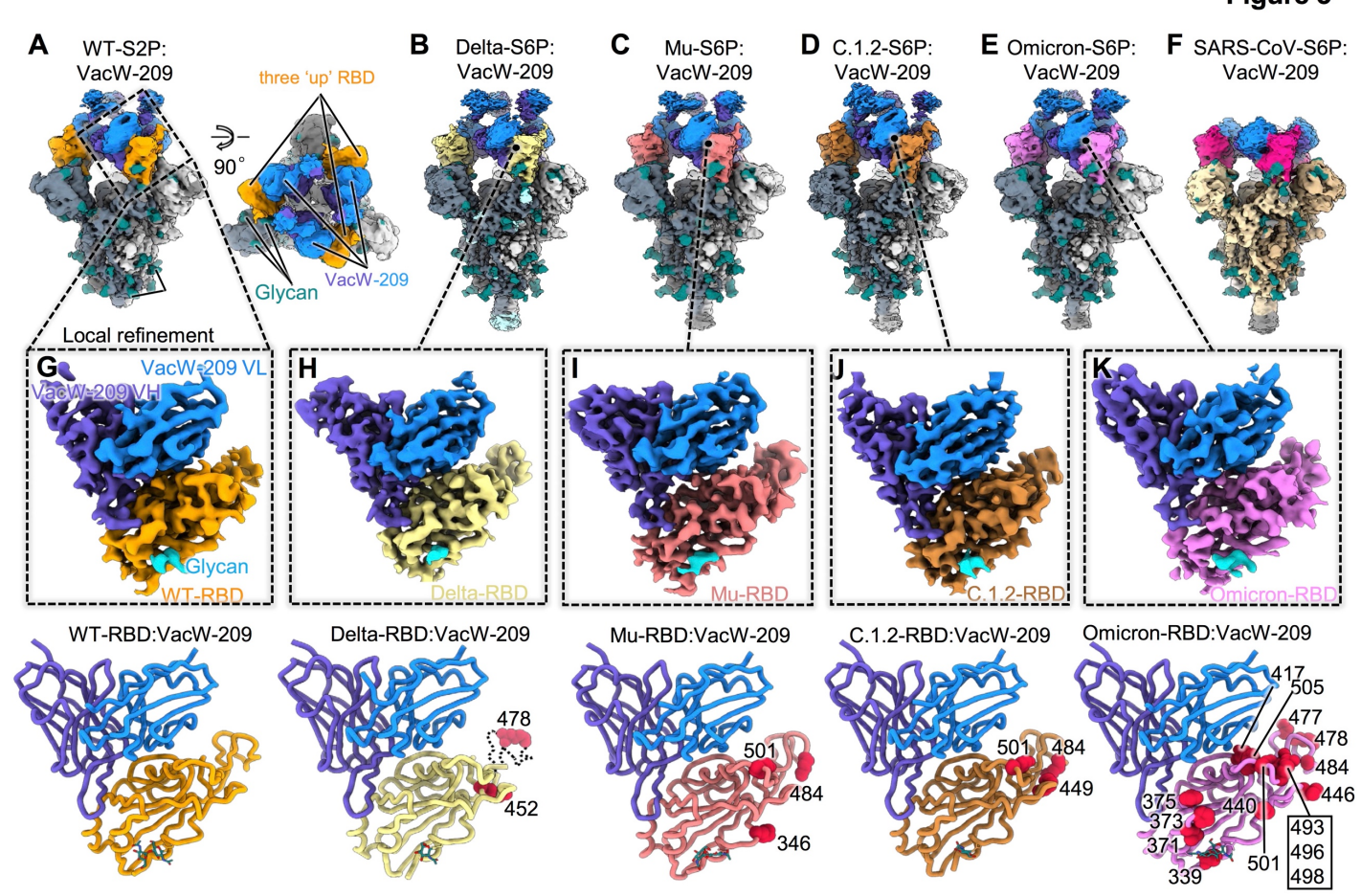

$\mathbf{L}$
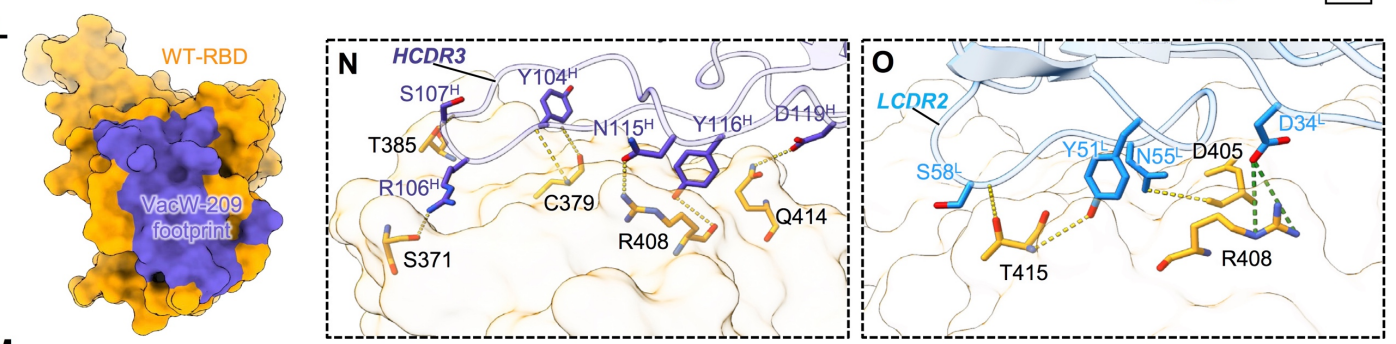

M
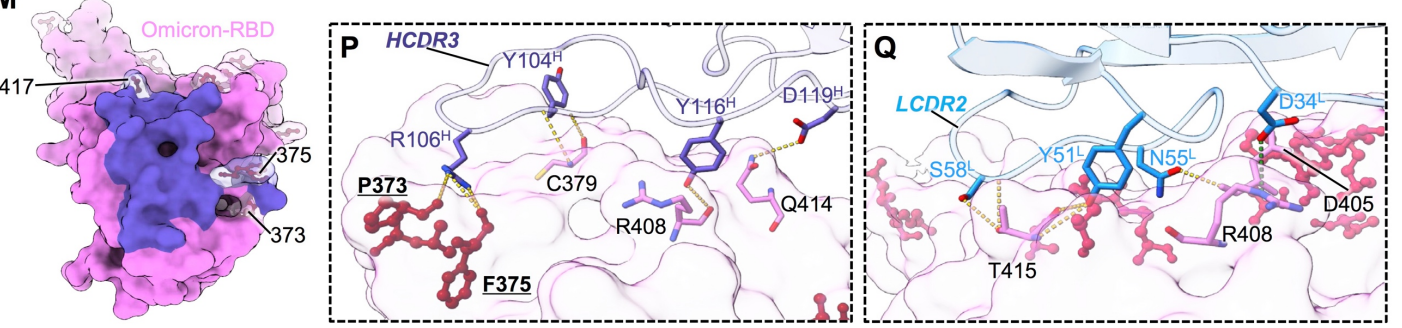

Figure 3. Cryo-EM structures of VacW-209 complexed with spike proteins of WT SARS-CoV-2, variants, and SARS-CoV.

449 (A-F) Cryo-EM density maps of VacW-209 in complex with spike proteins of

450 WT-S2P (A), Delta-S6P (B), Mu-S6P (C), C.1.2-S6P (D), Omicron-S6P (E), and

451 SARS-CoV-S6P (F). (G-K) Cryo-EM density maps (upper) and corresponding 452 atomic models (lower) of local refinement of VacW-209 in complex with WT453 RBD (G), Delta-RBD (H), Mu-RBD (I), C.1.2-RBD (J), and Omicron-RBD (K). 454 Models are represented as cartoon and key mutations on RBD are highlighted 455 as red balls. (L, M) The binding footprints of VacW-209 (colored in purple) on 456 WT-RBD (orange surface representation) (L) and Omicron-RBD (pink surface 
457 representation) (M). The mutated residues are rendered as red sticks with 458 transparent surface representation on Omicron RBD and those involved in 459 VacW-209 interactions are labeled. (N, O) Interaction details between WT-RBD 460 and VacW-209 heavy chain (N) and light chain (O). (P, $\mathbf{Q})$ Interaction details 461 between Omicron-RBD and VacW-209 heavy chain (P) and light chain (Q). 462 Hydrogen bonds and salt-bridges are labeled as orange and dark green dotted 463 lines respectively. 

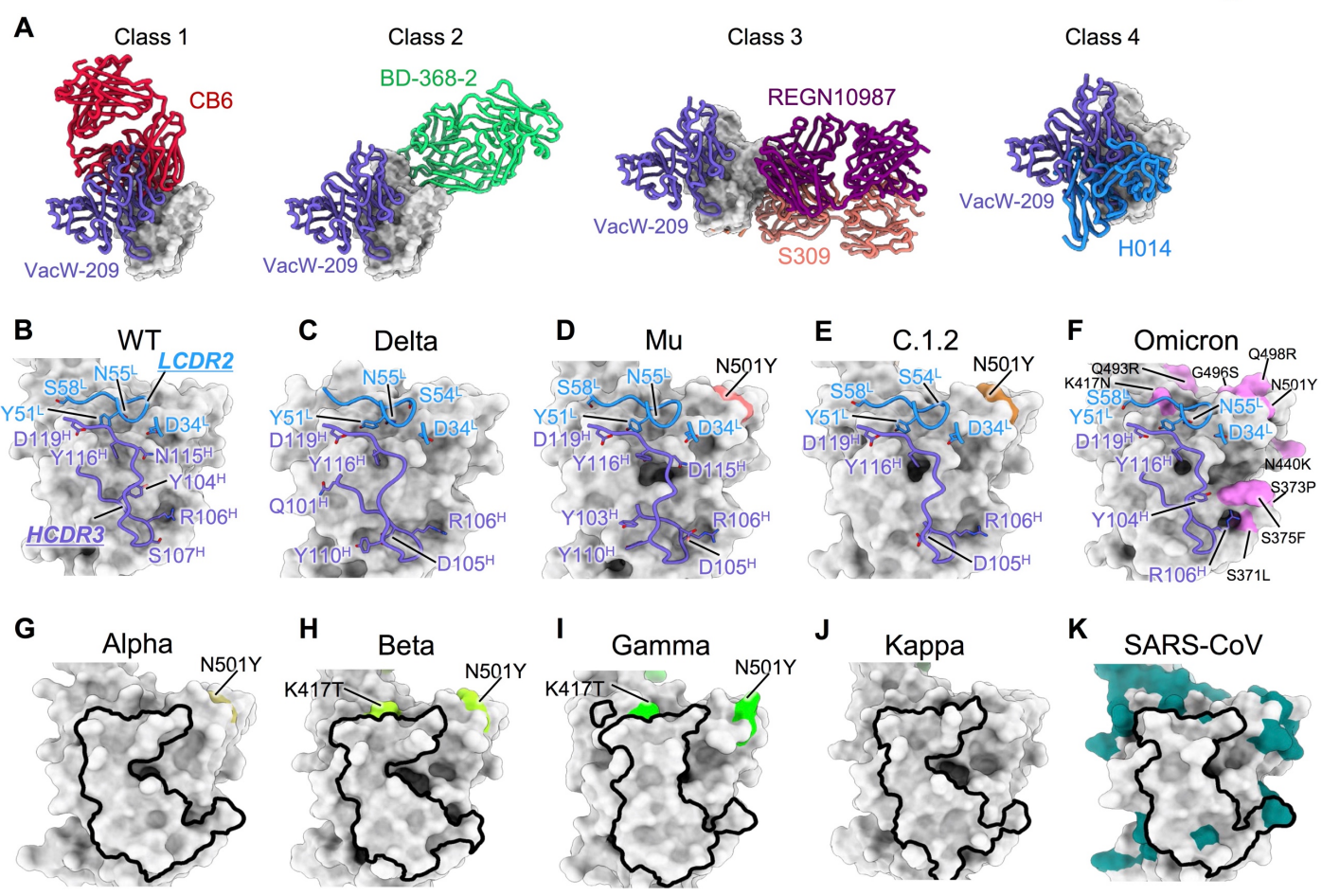

$\mathbf{L}$
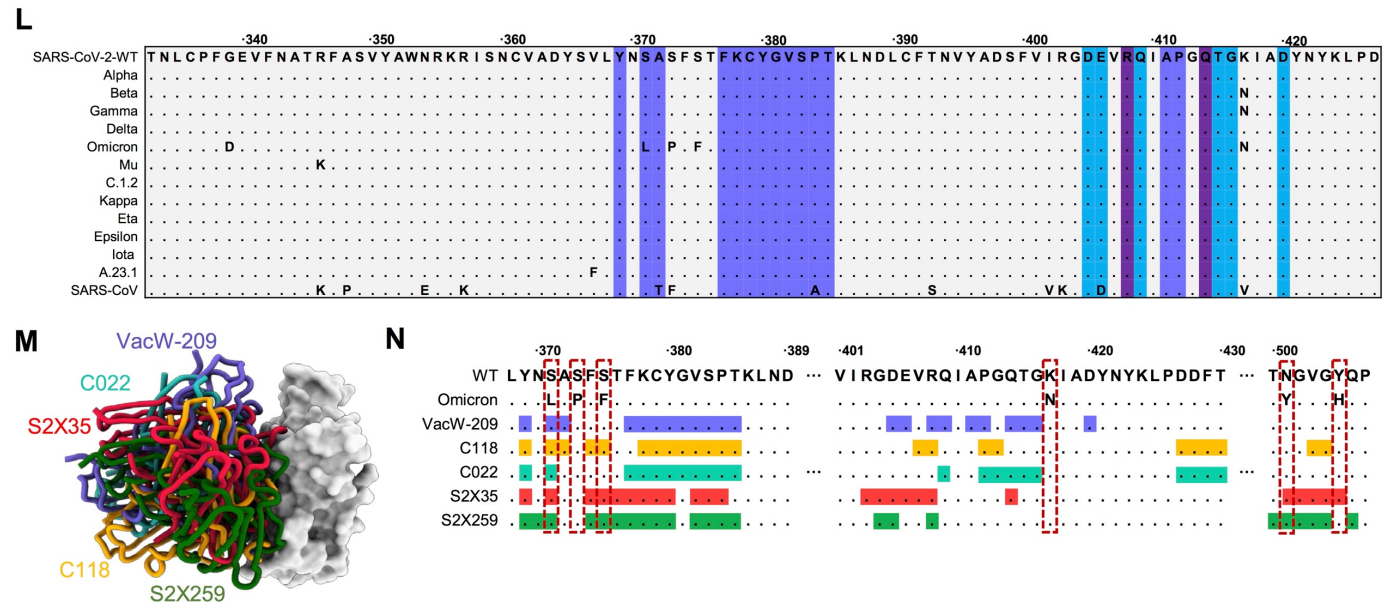

465 Figure 4. Binding mode and the epitope conservation of VacW-209.

466 (A) Comparison of the binding mode of VacW-209 to representative Class 1-4 467 nAbs. Class 1: CB6 (7C01), Class 2: BD-368-2 (7CHH), Class 3: REGN10987 468 (6XDG) and S309 (7R6W), and Class 4: H014 (7CAH). (B-F) Structural 469 compassion of VacW-209 bound to SARS-CoV-2 WT-RBD (B), Delta-RBD (C), 470 Mu-RBD (D), C.1.2-RBD (E), and Omicron-RBD (F). (G-K) Structure 471 comparison of RBDs of Alpha (G) (7LWV), Beta (H) (7VX1), Gamma (I) (7M8K), 472 Kappa (J) (7VXE) variants, and SARS-CoV (K) (7JN5). RBDs are shown as 473 gray surface representations and key mutations on RBDs are labeled. The 474 modeled VacW-209 footprints are shown on (G-K) based on the epitope 
475 information revealed in SARS-CoV-2 WT-S. (L) RBD sequences of SARS-CoV4762 WT and its 12 variants as well as SARS-CoV with highlighted footprint of 477 VacW-209 (slate blue: heavy chain, dodger blue: light chain, dark blue: both 478 chains). (M) VacW-209-like nAbs and their binding modes on RBD. VacW-209, 479 C118 (7RKS), C022 (7RKU), S2X35 (7R6W), and S2X259 (7M7W) are shown 480 as sticks and colored in blue, orange, cyan, red, and green, respectively. (N) 481 RBD sequence of SARS-CoV-2 WT and Omicron variant with highlighted 482 footprints of VacW-209, C118, C022, S2X35, and S2X259. Amino acids 483 substitutions revealed on Omicron variant are boxed. 


\section{Supplementary information}

3 Materials and Methods

\section{Study approval and plasma samples}

This study was approved by the Ethics Committee of Shenzhen Third People's Hospital, China (approval number: 2020-084). All participants had provided written informed consent for sample collection and subsequent analysis. All plasma samples were collected from 19 convalescent patients at Month 6 post recovery from the early COVID-19 pandemic. All plasma samples were stored at $-80{ }^{\circ} \mathrm{C}$ in the Biobank of Shenzhen Third People's Hospital and heat-

\section{Expression and purification of monoclonal neutralizing antibodies}

Gene sequences of published neutralizing antibodies (nAbs) downloaded from the National Center of Biotechnology Information (NCBI) were synthesized and cloned into the human full-length lgG1 expression vectors by Sangon Biotech and GenScript. Their protein data bank (PDB) codes were CB6 (7C01), REGN10933 (6XDG), CC12.1 (6XC2), BD-368-2 (7CHH), P2B-2F6 (7BWJ), C144 (7K90), REGN10987 (6XDG), C110 (7K8V), S309 (6WPS), H014 (7CAI), EY6A (6ZCZ), S304 (7JW0), P2C-1F11 (7CDI), C135 (7K8Z), S2A4 (7JVC), COVA1-16 (7JMV), C118 (7RKS), C022 (7RKU), S2X35 (7R6W), and S2X259 (7RAL), respectively. Paired heavy-chain and light-chain plasmids were cotransfected into $293 \mathrm{~F}$ cells. Monoclonal antibodies (mAbs) were purified from cell supernatants after five days using protein A columns according to the manufacturer's instructions (National Engineering Research Center for Biotechnology). Purified antibodies were quantified by NanoDrop and stored at $4{ }^{\circ} \mathrm{C}$ before use. Nine monoclonal nAbs (VacW-89, VacW-92, VacW-105, VacW-120, VacW-138, VacW-201, VacW-209, VacW-212, and VacW-215) were isolated from 2 individuals who received two doses of SARS-CoV-2 inactivated vaccines (WIBP-CorV, the Sinopharm COVID-19 vaccine, Wuhan Institute of Biological 
variants have been reported in our previous study(1). Here, we further tested their neutralizing activities against Beta, Mu, Omicron, and other variants.

\section{Incidence analysis of SARS-CoV-2 variants}

Daily numbers of collected SARS-CoV-2 sequences in the world in 2021 were obtained from the GISAID (https://www.gisaid.org/) including Beta, Delta, Mu, C.1.2, Omicron, and other variants. Graphs were plotted using GraphPad Prism 9 software.

\section{SARS-CoV-2 and SARS-CoV pseudoviruses}

Pseudovirus was generated by co-transfection of HEK-293T cells with a spikeexpressing plasmid and an env-deficient HIV-1 backbone vector (pNL43.Luc.R-E-) as previously described(1-3). Two days post co-transfection, the culture supernatant was harvested, clarified by centrifugation, filtered, and stored at $-80^{\circ} \mathrm{C}$. The infectious titer was measured by luciferase activity in the HEK-293T-hACE2 cells using Bright-Lite Luciferase reagent (Vazyme Biotech).

Detailed sequence information of spike protein was listed below, respectively.

SARS-CoV-2 wild-type (WT):

Wuhan-Hu-1, accession number: NC_045512;

\section{SARS-CoV-2 Beta:}

D80A, D251G, 242-243del, K417N, E484K, N501Y, D614G, A701V;

\section{SARS-CoV-2 Delta:}

T19R, G142D, 157-158del, L452R, T478K, D614G, P681R, D950N;

\section{SARS-CoV-2 Mu:}

T95I, Y144S, Y145N, R346K, E484K, N501Y, D614G, P681H, D950N;

\section{SARS-CoV-2 C.1.2:}

0 P9L, C136F, 144-145del, R190S, D215G, Y449H, E484K, N501Y, D614G, 1 H655Y, N679K, T761I, N859N;

2 SARS-CoV-2 Omicron:

3 A67V, 69-70del, T95I, G142D, 143-145del, N211I, 212del, 215EPEins, G339D, 4 S371L, S373P, S375F, K417N, N440K, G446S, S477N, T478K, E484A, Q493R, 5 G496S, Q498R, N501Y, Y505H, T547K, D614G, H655Y, N679K, P681H, 6 N764K, D796Y, N856K, Q954H, N969K, L981F; 
SARS-CoV wild-type (WT):

CUHK-W1, accession number: AAP13567.1.

Recombinant receptor binding domain (RBD) and trimeric spike proteins

The SARS-CoV-2 WT RBD protein (residues 319-541) was expressed with a His tag at the C-terminus. The RBD expression vectors of Mu and C.1.2 were constructed by point mutation based on the pCMV3-WT-RBD-His (Sino Biological, VG40592-CH). These RBD plasmids were transiently transfected into $293 \mathrm{~F}$ cells, respectively. After 5 days, RBD proteins were collected from the supernatant using Ni-sepharose fast-flow 6 resin (GE Healthcare) and eluted by $250 \mathrm{mM}$ imidazole. The RBD proteins of SARS-CoV-2 Omicron (40592-V08H121) and SARS-CoV (40150-V08B2) were purchased from Sino Biological. All RBD proteins were used to measure the binding affinity of mAbs. The SARS-CoV-2 WT S-2P protein (residues 1-1208) was prepared in our previous study $(4,5)$, carrying two stabilizing Pro substitutions (986 and 987) and "GSAS" substitutions at the furin cleavage site (682-685). The other S-6P proteins of SARS-CoV-2 Delta, Mu, C.1.2, and SARS-CoV were expressed and purified in this study. Compared with S-2P, S-6P version contained additional four Pro substitutions (F817P, A892P, A899P, and A942P). The coding genes of spike ectodomain followed by a foldon trimerization motif, a His tag, and a flag tag at the C-terminus were synthesized and cloned into the pcDNA3.4 vector by GenScript. All S-6P plasmids were transiently transfected into $293 \mathrm{~F}$ cells, respectively. After 5 days, the secreted S-6P proteins were purified from the supernatant with Ni-sepharose fast-flow 6 resin (GE Healthcare) and 250 $\mathrm{mM}$ imidazole. The SARS-CoV-2 Omicron S-6P protein (40589-V08H26) was purchased from Sino Biological. All S-2P or S-6P proteins mentioned here were further used to perform the cryo-electron microscopy (Cryo-EM) experiment.

\section{Pseudovirus-based neutralizing assay}

96 To determine the neutralizing activity, plasma samples or purified mAbs were serially diluted and then incubated with an equal volume of pseudovirus at $37^{\circ} \mathrm{C}$ for $1 \mathrm{~h}$. HEK-293T-hACE2 cells were subsequently added to the 96-well plates. After a 48-h incubation, the culture medium was removed, and $100 \mu \mathrm{L}$ of Bright- 
100 Lite Luciferase reagent was added to the cells. After an incubation at RT for 2 101 mins, $90 \mu \mathrm{L}$ of cell lysate was transferred to the 96-well white solid plates for 102 measurements of luminescence using Varioskan ${ }^{\mathrm{TM}}$ LUX multimode microplate 103 reader (Thermo Fisher Scientific). The 50\% inhibitory dilution (ID 50 ) for plasma 104 or $50 \%$ inhibitory concentration $\left(\mathrm{IC}_{50}\right)$ for $\mathrm{mAbs}$ was calculated using GraphPad 105 Prism 9 software by log (inhibitor) vs. normalized response - Variable slope 106 (four parameters) model.

\section{Binding analysis by surface plasmon resonance (SPR)}

109 The binding assays of mAbs to the RBD proteins were performed using the 110 Biacore 8K system (GE Healthcare). Specifically, one flow cell of the CM5 111 sensor chips were covalently coated with the RBD proteins in $10 \mathrm{mM}$ sodium 112 acetate buffer ( $\mathrm{pH}$ 5.0) for a final RU (response units) around 250, whereas the 113 other flow cell was left uncoated and blocked as a control. All the assays were 114 run at a flow rate of $30 \mu \mathrm{L} / \mathrm{min}$ in HBS-EP buffer (10 mM HEPES pH 7.4, 150 $115 \mathrm{mM} \mathrm{NaCl}, 3 \mathrm{mM}$ EDTA, and 0.05\% Tween-20). Serially diluted mAbs were 116 injected for $60 \mathrm{~s}$ respectively and the resulting data were fit in a 1:1 binding 117 model with Biacore Evaluation software (GE Healthcare). Every measurement 118 was performed two times and the individual values were used to produce the 119 mean affinity constant.

\section{Competition enzyme linked immunosorbent assay (ELISA)}

122 The SARS-CoV-2 WT RBD protein was coated into 96 -well plates at $4{ }^{\circ} \mathrm{C}$ 123 overnight. The plates were washed with PBST buffer and blocked with $5 \%$ skim 124 milk and $2 \%$ bovine albumin in PBS at RT for $1 \mathrm{~h}$. Human ACE2 (Sino Biological, 125 10108-H08H) or VacW-209 coupled with HRP (Abcam) were mixed with serially 126 diluted mAbs, added into the plates, and then incubated at $37^{\circ} \mathrm{C}$ for $1 \mathrm{~h}$. The 127 TMB substrate (Sangon Biotech) was added and incubated at RT for 20 mins 128 and the reaction was stopped by $2 \mathrm{M} \mathrm{H}_{2} \mathrm{SO}_{4}$. The readout was detected at a 129 wave length of $450 \mathrm{~nm}$. The tested mAbs were 3-fold serially diluted from 10 $130 \mu \mathrm{g} / \mathrm{mL}$. VRC01 is a HIV-1-specific antibody and used here as a negative control, 131 which is considered as no competition with any SARS-CoV-2-specific mAbs. 132 The percentage of competition was calculated by the formula: $\left(1-\mathrm{OD}_{450}\right.$ of 
133 tested $\mathrm{mAbs} / \mathrm{OD}_{450}$ of $\mathrm{VRC0} 1$ control) $\times 100 \%$, and $50 \%$ was set as the cutoff

134 indicating an obvious competition.

\section{Cryo-EM sample preparation and data collection}

137 Aliquots $(3 \mu \mathrm{L})$ of $3.5 \mathrm{mg} / \mathrm{mL}$ mixtures of purified SARS-CoV-2 WT or variant or

138 SARS-CoV spike proteins in complex with excess Fab fragments of VacW-209 139 were incubated in $0.01 \%$ (v/v) Digitonin (Sigma) and then loaded onto glow140 discharged (60 s at $20 \mathrm{~mA}$ ) holey carbon Quantifoil grids (R1.2/1.3, 200 mesh, 141 Quantifoil Micro Tools) using a Vitrobot Mark IV (ThermoFisher Scientific) at $142100 \%$ humidity and $4{ }^{\circ} \mathrm{C}$. Data were acquired using the SerialEM software on 143 an FEI Tecnai F30 transmission electron microscope (ThermoFisher Scientific) 144 operated at $300 \mathrm{kV}$ and equipped with a Gatan K3 direct detector. Images were 145 recorded in the 36-frame movie mode at a nominal 39,000× magnification at 146 super-resolution mode with a pixel size of $0.339 \AA$. The total electron dose was 147 set to $60 \mathrm{e}^{-} \AA^{-2}$ and the exposure time was $4.5 \mathrm{~s}$.

\section{Cryo-EM data processing}

150 Drift and beam-induced motion correction was performed with MotionCor2(6) 151 to produce a micrograph from each movie. Contrast transfer function (CTF) 152 fitting and phase-shift estimation were conducted with $\operatorname{Gctf}(7)$. Micrographs with 153 astigmatism, obvious drift, or contamination were discarded before 154 reconstruction. The following reconstruction procedures were performed by 155 using Cryosparc V3(8). In brief, particles were automatically picked by using 156 the "Blob picker" or "Template picker". Several rounds of reference-free 2D 157 classifications were performed and the selected good particles were then 158 subjected to ab-initio reconstruction, heterogeneous refinement and final non159 uniform refinement. The resolution of all density maps was determined by the 160 gold-standard Fourier shell correlation curve, with a cutoff of 0.143(9). Local 161 map resolution was estimated with ResMap(10).

\section{Cryo-EM model building and analysis}

164 The initial model of nAbs were generated from homology modeling by Accelrys 165 Discovery Studio software (available from: URL: https://www.3dsbiovia.com). 
166 The structure of RBD from the structure of WT trimeric spike (6VSB(11)) was 167 used as the initial modes of our WT-RBD and Omicron RBD. We initially fitted 168 the templates into the corresponding final cryo-EM maps using Chimera(12), 169 and further corrected and adjusted them manually by real-space refinement in 170 Coot(13). The resulting models were then refined with 171 phenix.real_space_refine in PHENIX(14). These operations were executed 172 iteratively until the problematic regions, Ramachandran outliers, and poor 173 rotamers were either eliminated or moved to favored regions. The final atomic 174 models were validated with $\operatorname{Molprobity}(15,16)$. All figures were generated with 175 Chimera or ChimeraX $(17,18)$.

\section{References}

178 1. L. Cheng et al., Cross-neutralization of SARS-CoV-2 Kappa and Delta $179 \quad$ variants by inactivated vaccine-elicited serum and monoclonal antibodies. $180 \quad$ Cell Discov 7, 112 (2021).

181 2. B. Ju et al., Human neutralizing antibodies elicited by SARS-CoV-2 182 infection. Nature, (2020).

183 3. Q. Zhang et al., Potent and protective IGHV3-53/3-66 public antibodies 184 and their shared escape mutant on the spike of SARS-CoV-2. Nat $185 \quad$ Commun 12, 4210 (2021).

186 4. T. Li et al., Cross-neutralizing antibodies bind a SARS-CoV-2 cryptic site and resist circulating variants. Nat Commun 12, 5652 (2021).

5. T. Li et al., SARS-CoV-2 spike produced in insect cells elicits high neutralization titres in non-human primates. Emerg Microbes Infect $\mathbf{9}$, 2076-2090 (2020).

6. S. Q. Zheng et al., MotionCor2: anisotropic correction of beam-induced

194 7. K. Zhang, Gctf: Real-time CTF determination and correction. Journal of structural biology 193, 1-12 (2016).

8. A. Punjani, J. L. Rubinstein, D. J. Fleet, M. A. Brubaker, cryoSPARC: algorithms for rapid unsupervised cryo-EM structure determination. Nature methods 14, 290-296 (2017). 
199 9. S. H. Scheres, S. Chen, Prevention of overfitting in cryo-EM structure determination. Nature methods 9, 853-854 (2012).

10. A. Kucukelbir, F. J. Sigworth, H. D. Tagare, Quantifying the local resolution of cryo-EM density maps. Nature methods 11, 63-65 (2014).

11. D. Wrapp et al., Cryo-EM structure of the 2019-nCoV spike in the prefusion conformation. Science 367, 1260-1263 (2020).

12. E. F. Pettersen et al., UCSF Chimera--a visualization system for exploratory research and analysis. Journal of computational chemistry 25, 1605-1612 (2004).

13. P. Emsley, K. Cowtan, Coot: model-building tools for molecular graphics. Acta crystallographica. Section D, Biological crystallography 60, 2126-2132 (2004).

14. P. D. Adams et al., PHENIX: a comprehensive Python-based system for macromolecular structure solution. Acta crystallographica. Section D, Biological crystallography 66, 213-221 (2010).

15. V. B. Chen et al., MolProbity: all-atom structure validation for macromolecular crystallography. Acta crystallographica. Section D,

217 16. X. Robert, P. Gouet, Deciphering key features in protein structures with the new ENDscript server. Nucleic acids research 42, W320-324 (2014).

219 17. T. D. Goddard et al., UCSF ChimeraX: Meeting modern challenges in visualization and analysis. Protein Sci 27, 14-25 (2018).

221 18. E. F. Pettersen et al., UCSF ChimeraX: Structure visualization for researchers, educators, and developers. Protein Sci 30, 70-82 (2021). 

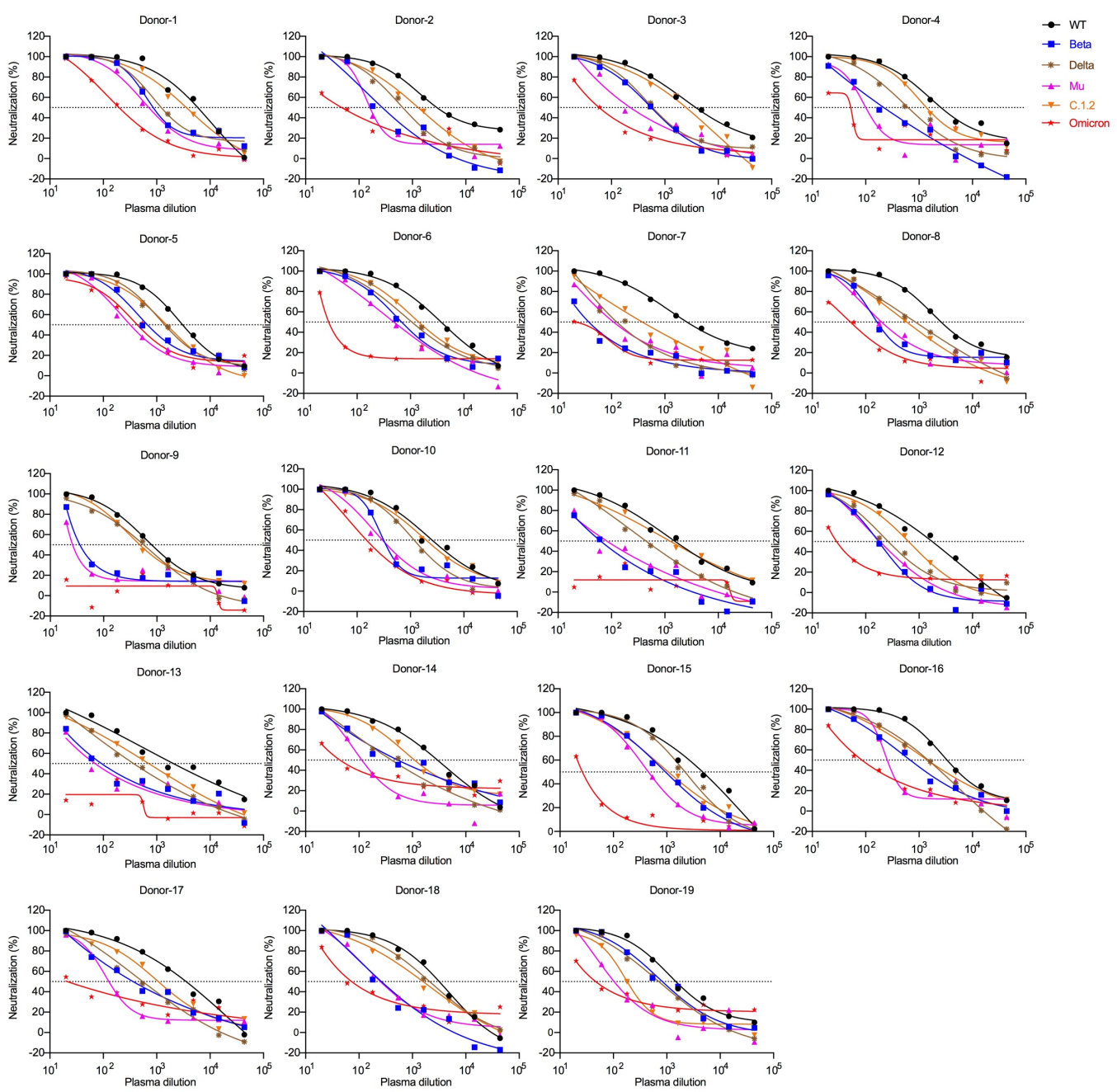

225 Figure S1. Neutralization curves of plasma samples from 19 convalescent 226 patients recovered from the first wave of COVID-19 pandemic against WT,

227 Beta, Delta, Mu, C.1.2, and Omicron pseudoviruses.

228 All plasma samples were serially 3 -fold diluted from $1: 20$. A $50 \%$ reduction in 229 viral infectivity was indicated by a horizontal dashed line. One out of two 230 independent experiments with similar results. 

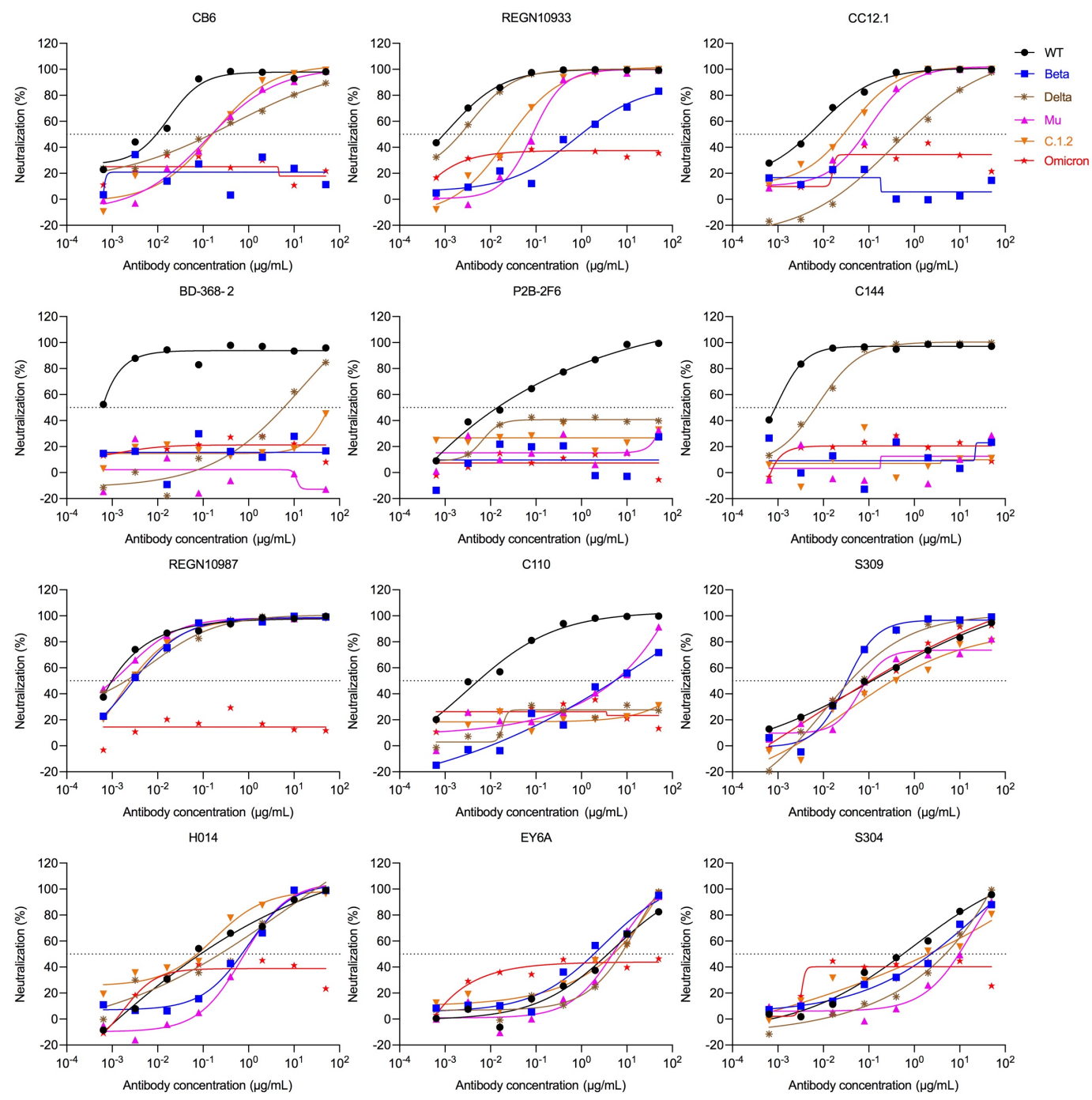

Figure S2. Neutralization curves of 12 representative nAbs against WT,

233 Beta, Delta, Mu, C.1.2, and Omicron pseudoviruses.

234 All nAbs were serially 5 -fold diluted from $50 \mu \mathrm{g} / \mathrm{mL}$. Class 1: CB6, REGN10933, 235 CC12.1; Class 2: BD-368-2, P2B-2F6, C144; Class 3: REGN10987, C110, 236 S309; Class 4: H014, EY6A, S304. A 50\% reduction in viral infectivity was 237 indicated by a horizontal dashed line. One out of two independent experiments 238 with similar results. 

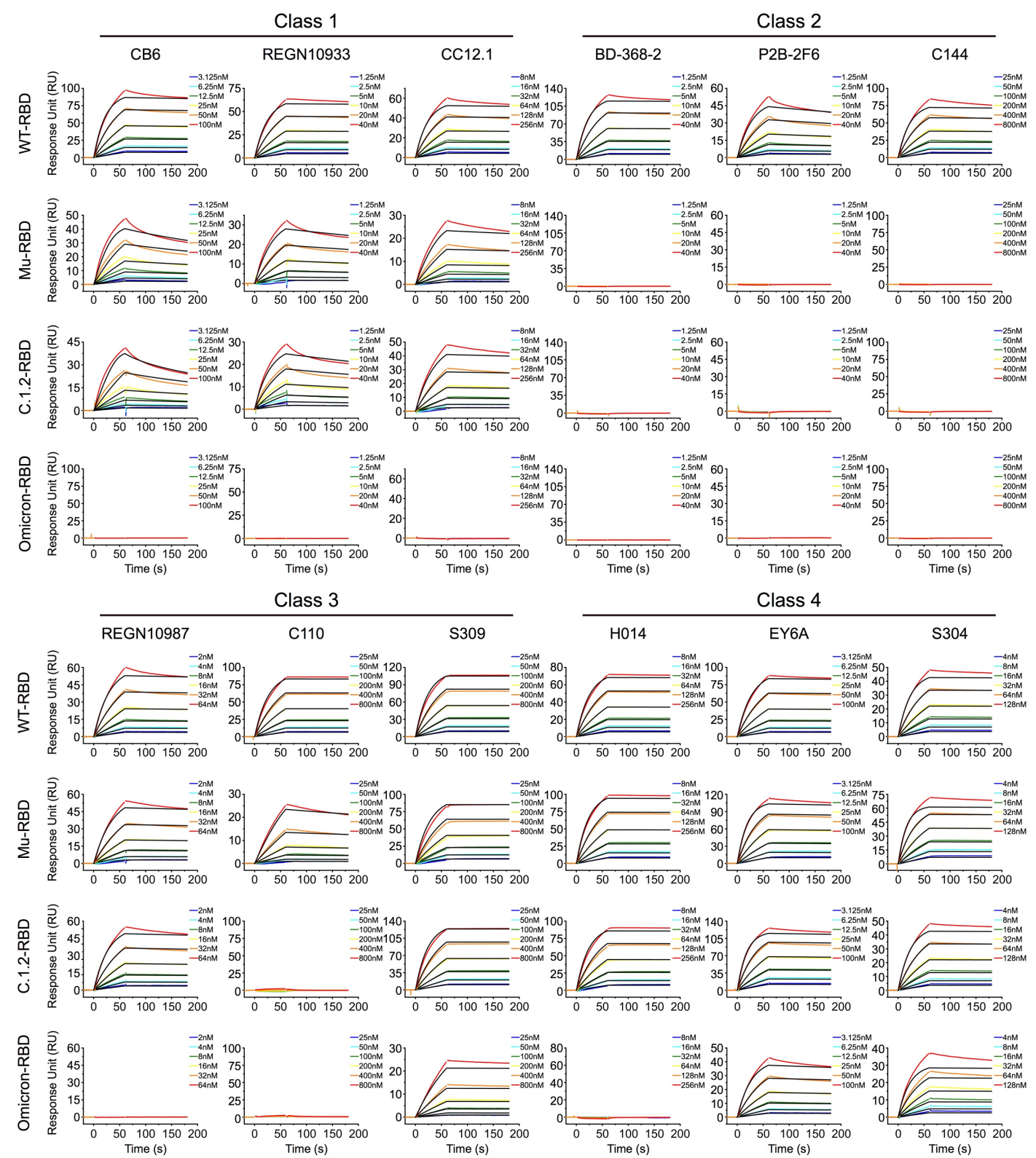

240 Figure S3. The binding affinities of 12 representative nAbs to SARS-CoV2412 WT, Mu, C.1.2, and Omicron RBD proteins measured by SPR.

242 One out of two independent experiments with similar results. 
A

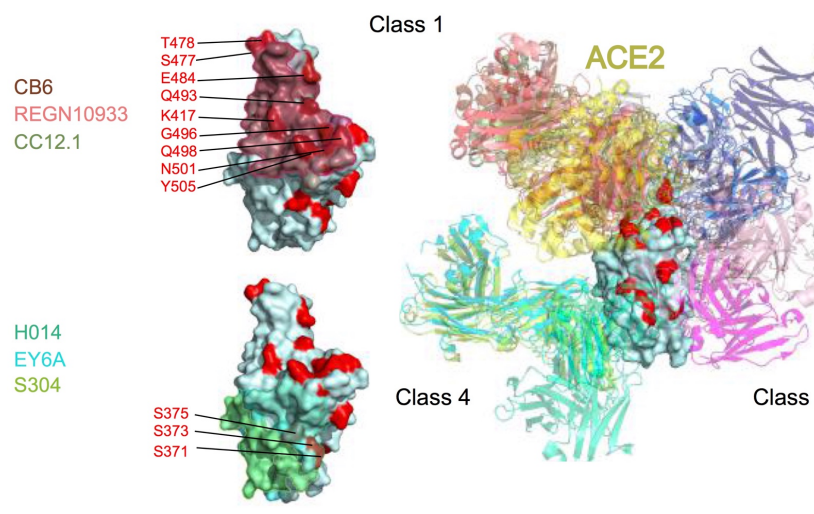

Class 2
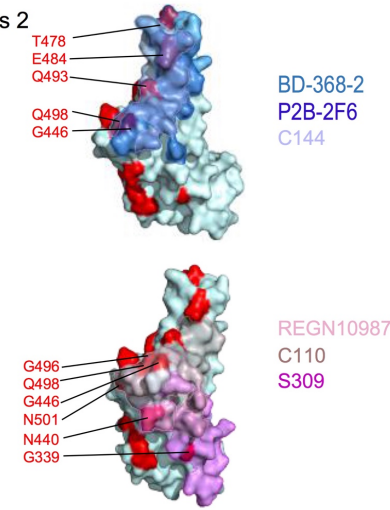

B

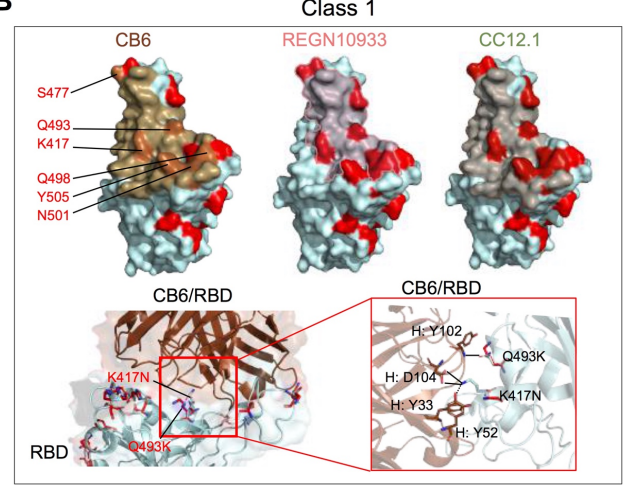

D

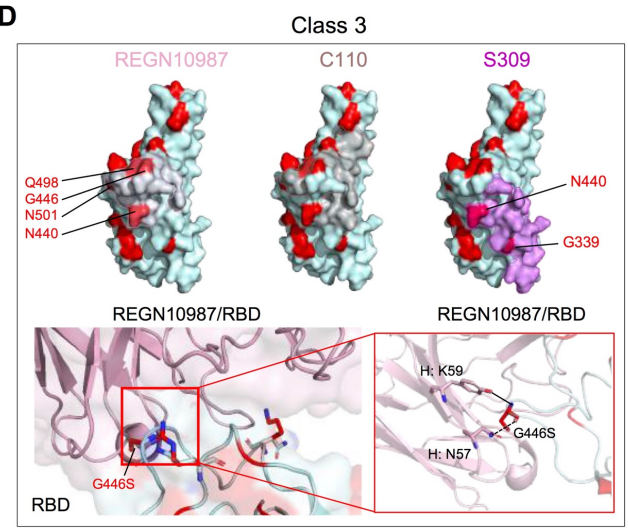

C

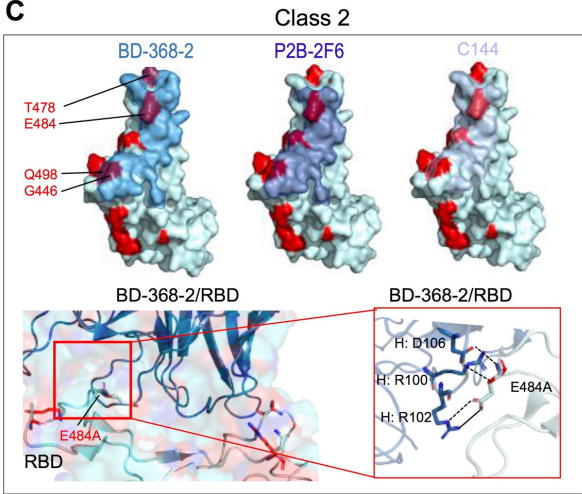

E

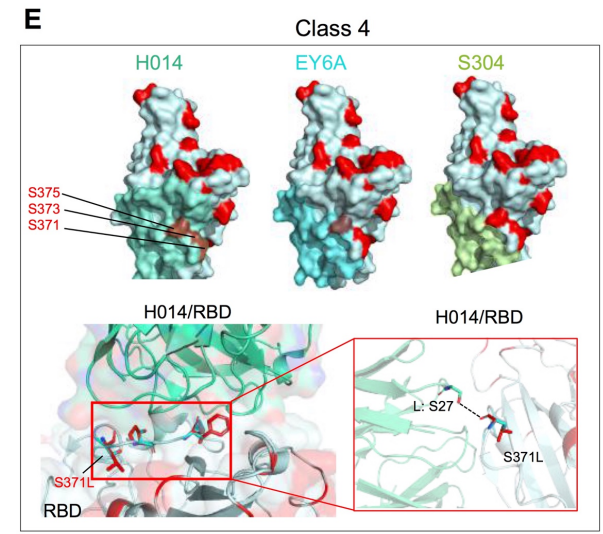

Figure S4. Structural analysis of 12 representative nAbs binding to SARS-

\section{CoV-2 RBD.}

246 (A) Overall structure of ACE2 (PDB: 7DMU) and 12 representative nAbs in 247 complex with SARS-CoV-2 RBD. Footprints of four classes of representative 248 nAbs were represented on the RBD in different colors. The mutated residues 249 appeared in their epitopes were shown in red and labeled beside. The structural 250 analysis of Omicron escaping from nAbs of Class 1 (B), Class 2 (C), Class 3 251 (D), and Class 4 (E). Cartoon diagrams showing the detailed interface between 252 nAbs and RBD. Hydrogen bond and salt bridge interactions were represented 253 by dashed and black lines, respectively. "H:" indicated antibody heavy chain. 254 "L:" indicated antibody light chain. 

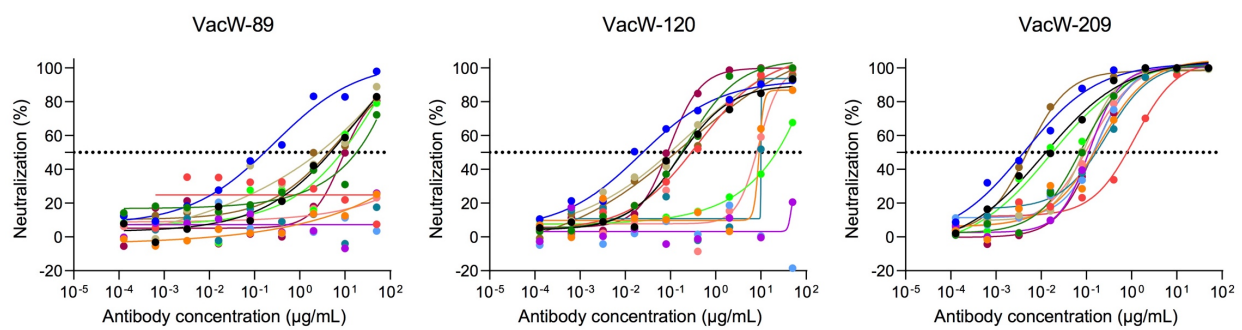

$\rightarrow$ WT

Antibody concentration $(\mu \mathrm{g} / \mathrm{mL})$

Antibody concentration $(\mu \mathrm{g} / \mathrm{mL})$

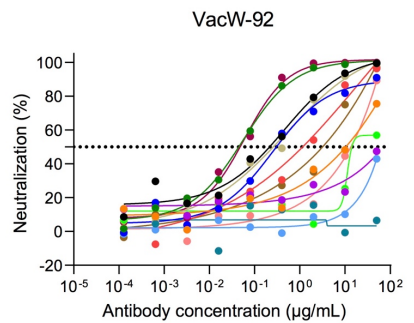

VacW-138
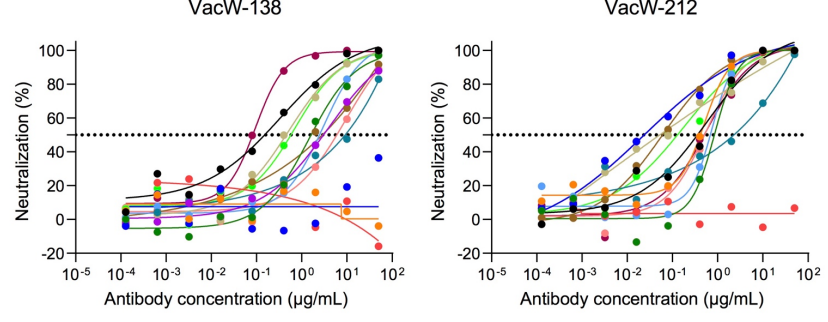

VacW-105

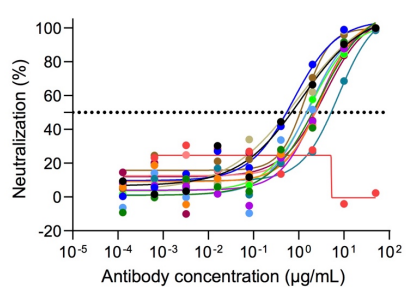

VacW-201
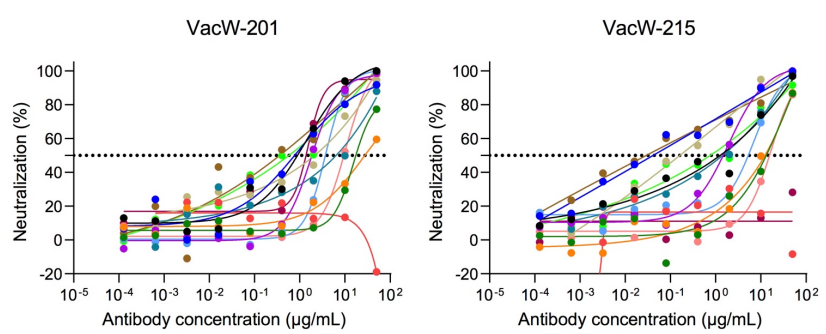

$\rightarrow$ Alpha

- Beta

- Gamma

- Delta

$\rightarrow \mathrm{Mu}$

$\rightarrow$ C.1.2

- Kappa

- Eta

$\rightarrow$ Epsilon

$\rightarrow$ lota
$\rightarrow$ A.23.1

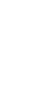

256 Figure S5. Neutralization curves of 9 nAbs isolated from 2 individuals who

257 received SARS-CoV-2 inactivated vaccines against WT, Alpha, Beta, Delta,

258 Mu, C.1.2, Omicron, and other mutated pseudoviruses.

259 All nAbs were serially 5 -fold diluted from $50 \mu \mathrm{g} / \mathrm{mL}$. A $50 \%$ reduction in viral

260 infectivity was indicated by a horizontal dashed line. One out of at least two 261 independent experiments with similar results. 

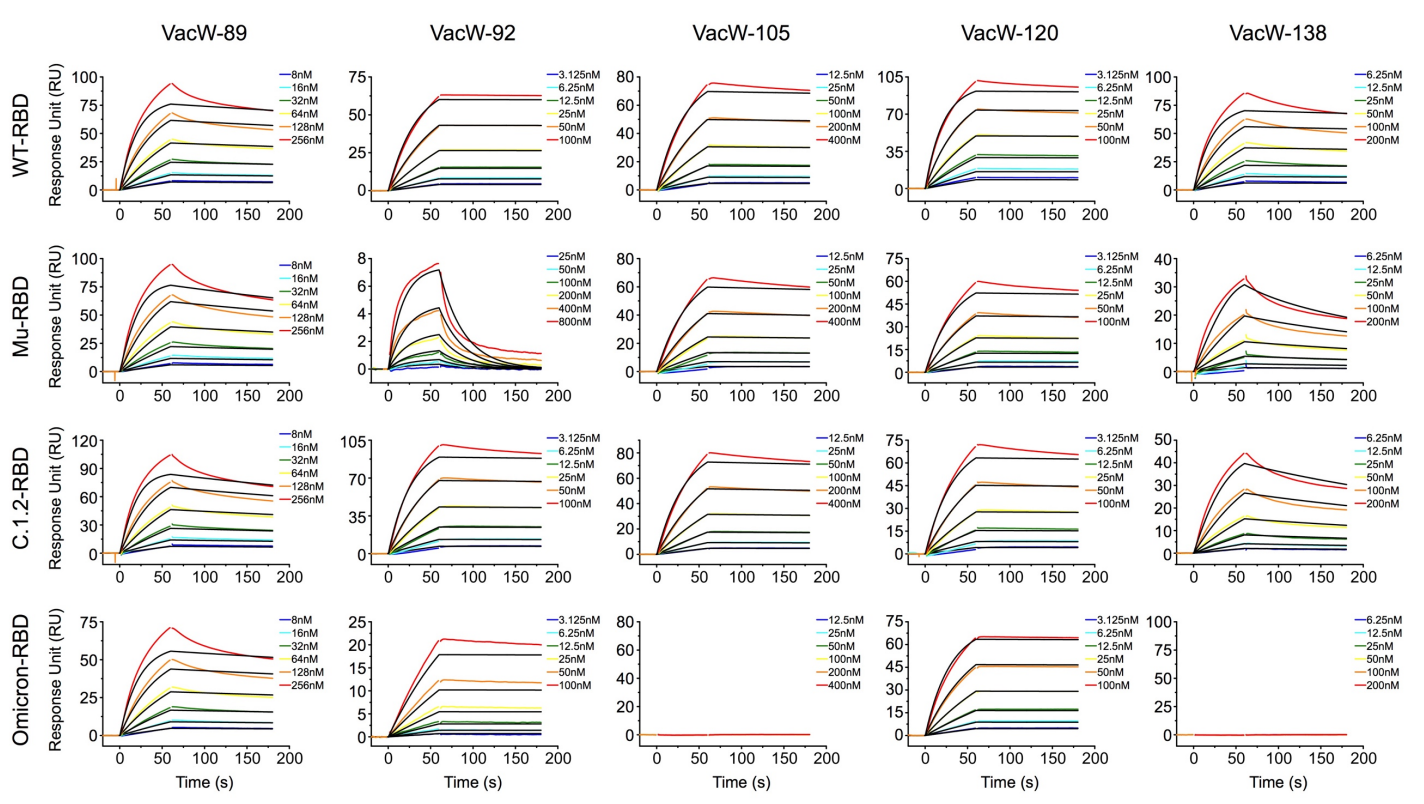

VacW-201

VacW-209

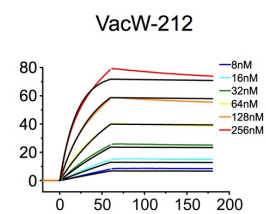

VacW-215
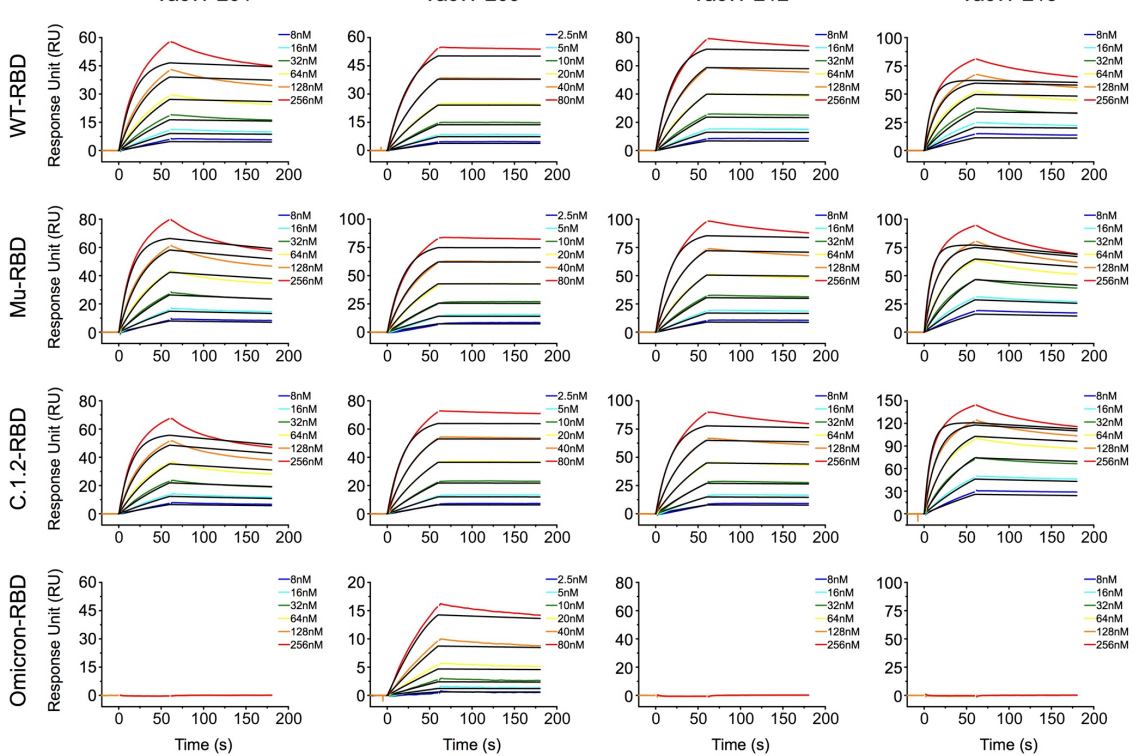

Figure S6. The binding affinities of $9 \mathrm{nAbs}$ isolated from 2 individuals who received SARS-CoV-2 inactivated vaccines to SARS-CoV-2 WT, Mu, C.1.2, and Omicron RBD proteins measured by SPR.

266 One out of two independent experiments with similar results. 


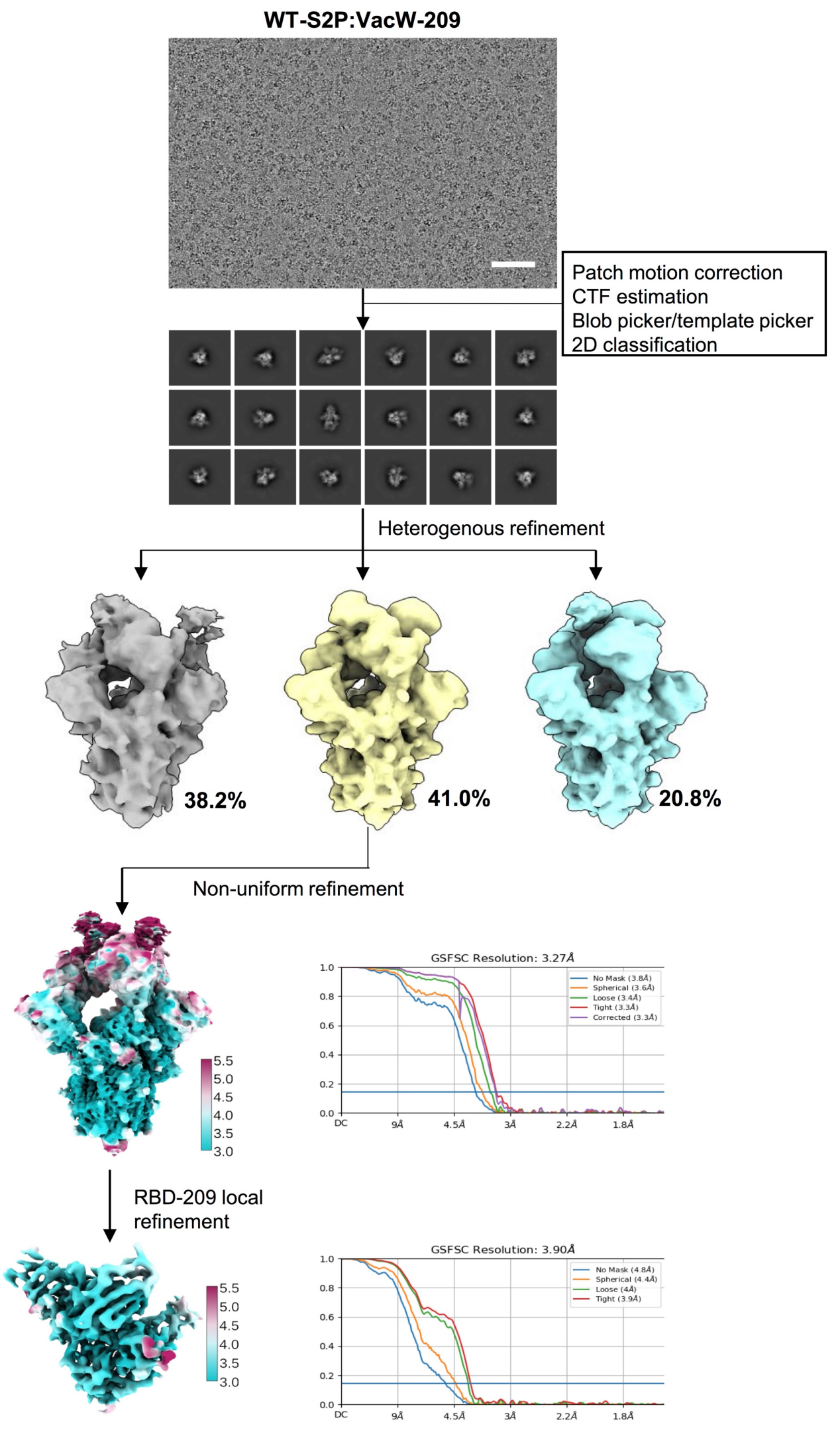

268 Figure S7. Single-particle cryo-EM images processing workflow and the 269 global and local resolution estimation for the immune complex of SARS270 CoV-2 WT-S2P:VacW-209. 


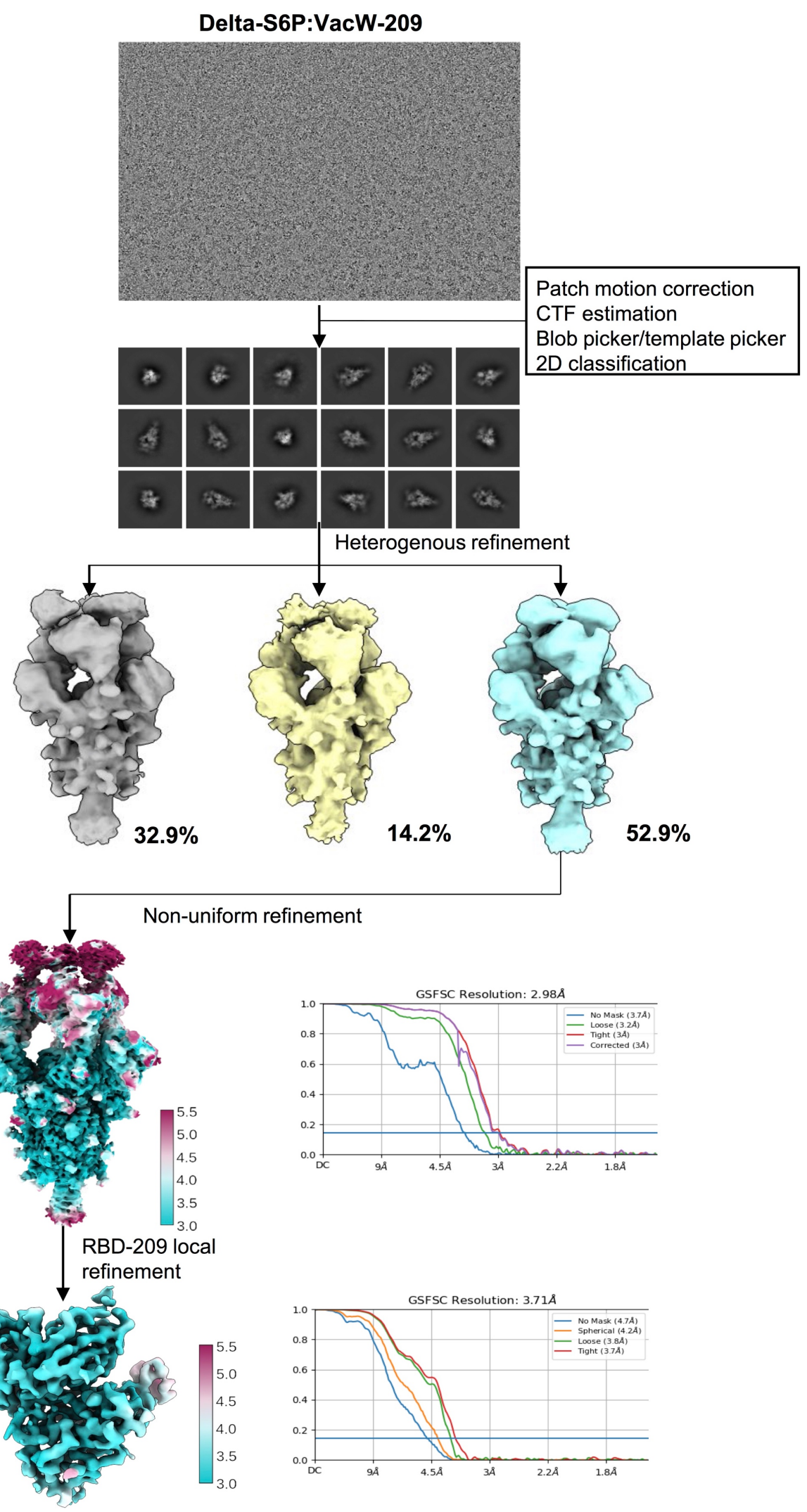

272 Figure S8. Single-particle cryo-EM images processing workflow and the 273 global and local resolution estimation for the immune complex of SARS274 CoV-2 Delta-S6P:VacW-209. 

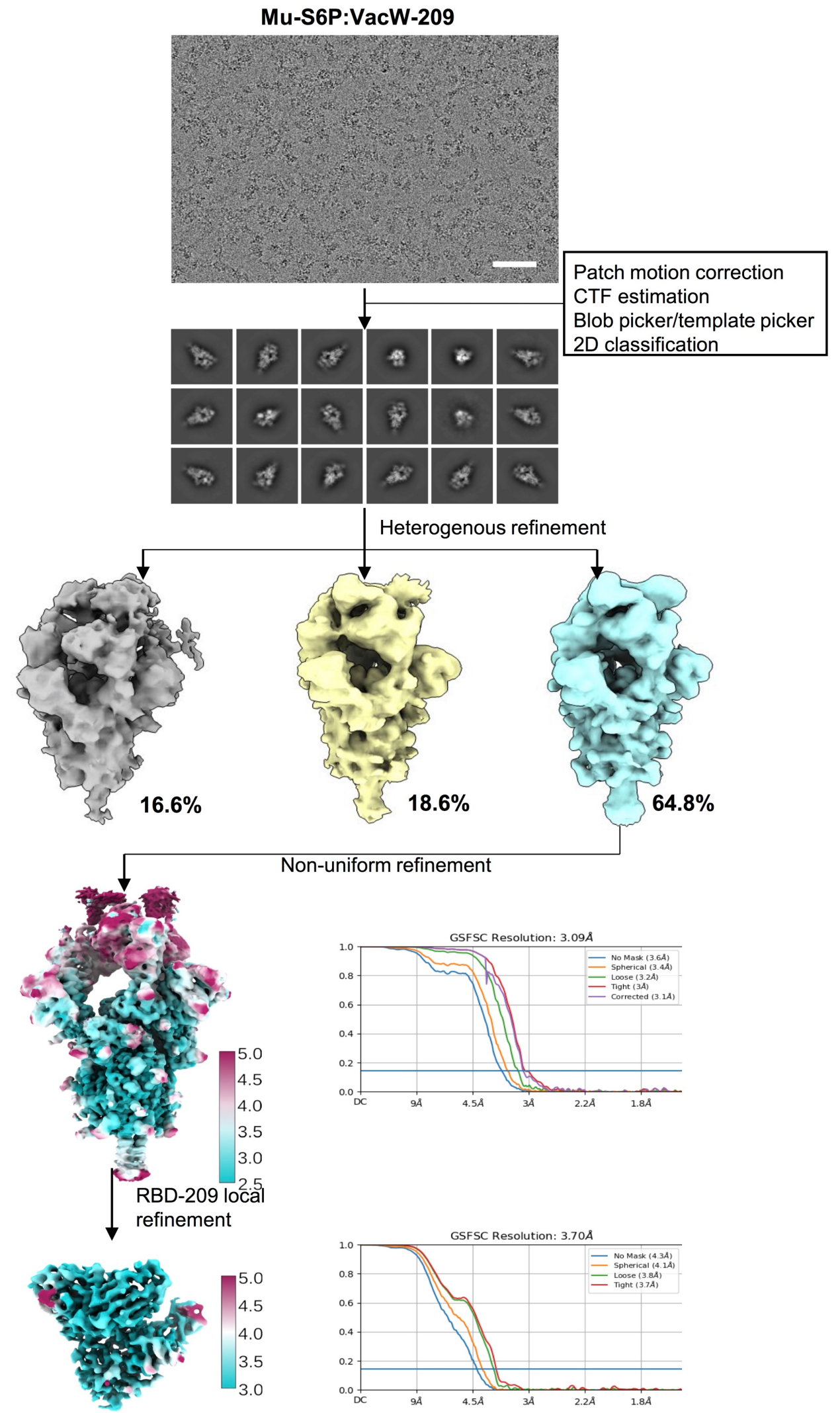

Non-uniform refinement

276 Figure S9. Single-particle cryo-EM images processing workflow and the

277 global and local resolution estimation for the immune complex of SARS-

278 CoV-2 Mu-S6P:VacW-209. 


\section{C.1.2-S6P:VacW-209}

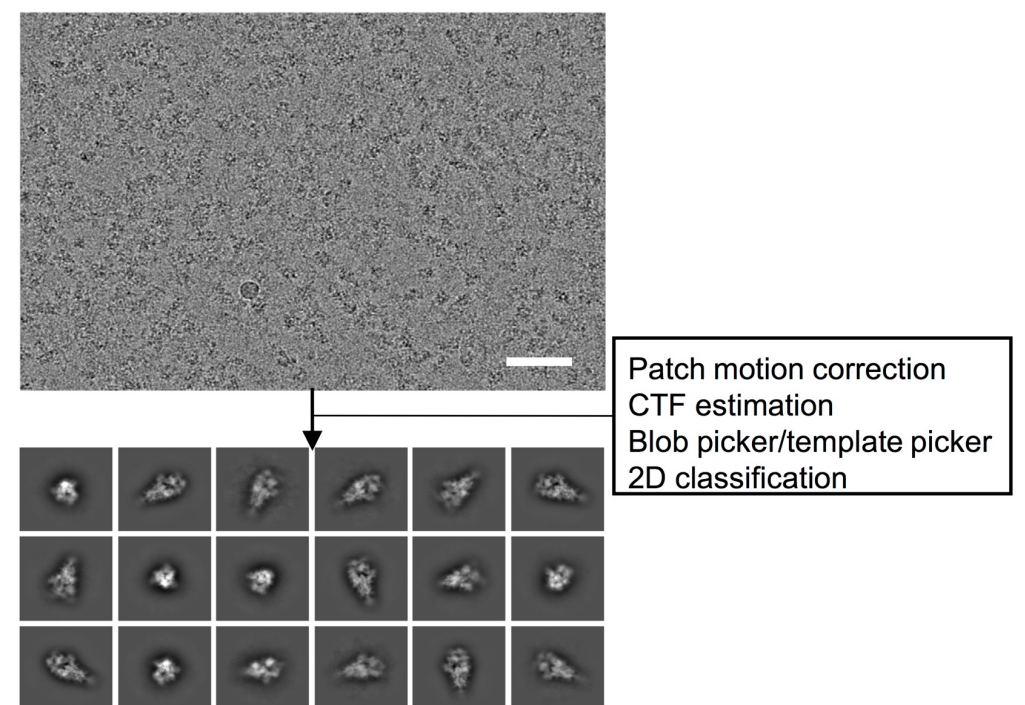

$26.1 \%$

$47.8 \%$

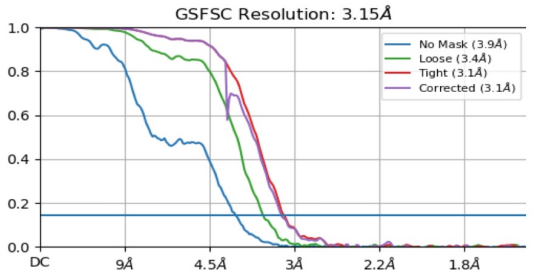

280 Figure S10. Single-particle cryo-EM images processing workflow and the 281 global and local resolution estimation for the immune complex of SARS282 CoV-2 C.1.2-S6P:VacW-209. 

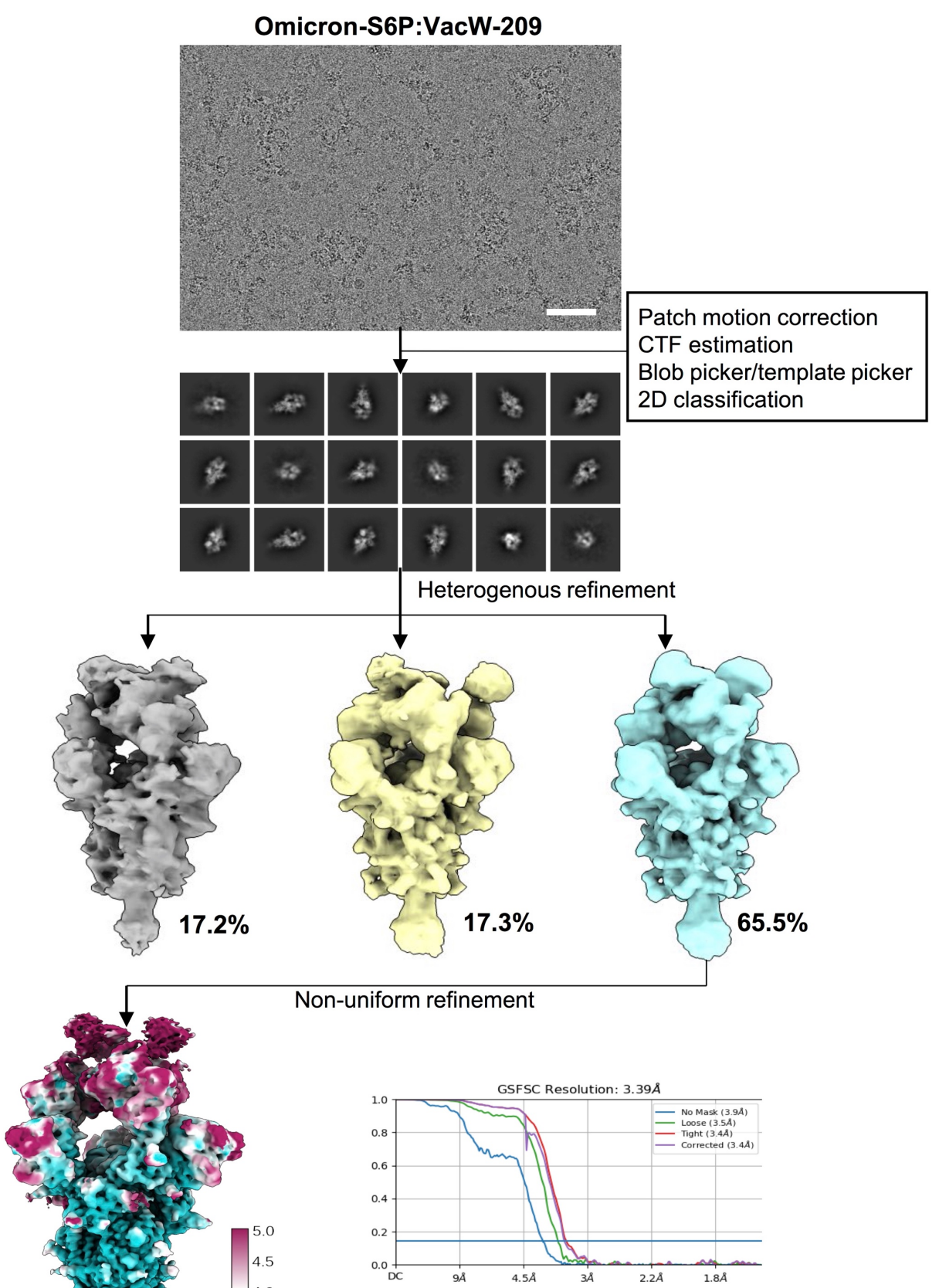

284 Figure S11. Single-particle cryo-EM images processing workflow and the 285 global and local resolution estimation for the immune complex of SARS286 CoV-2 Omicron-S6P:VacW-209. 


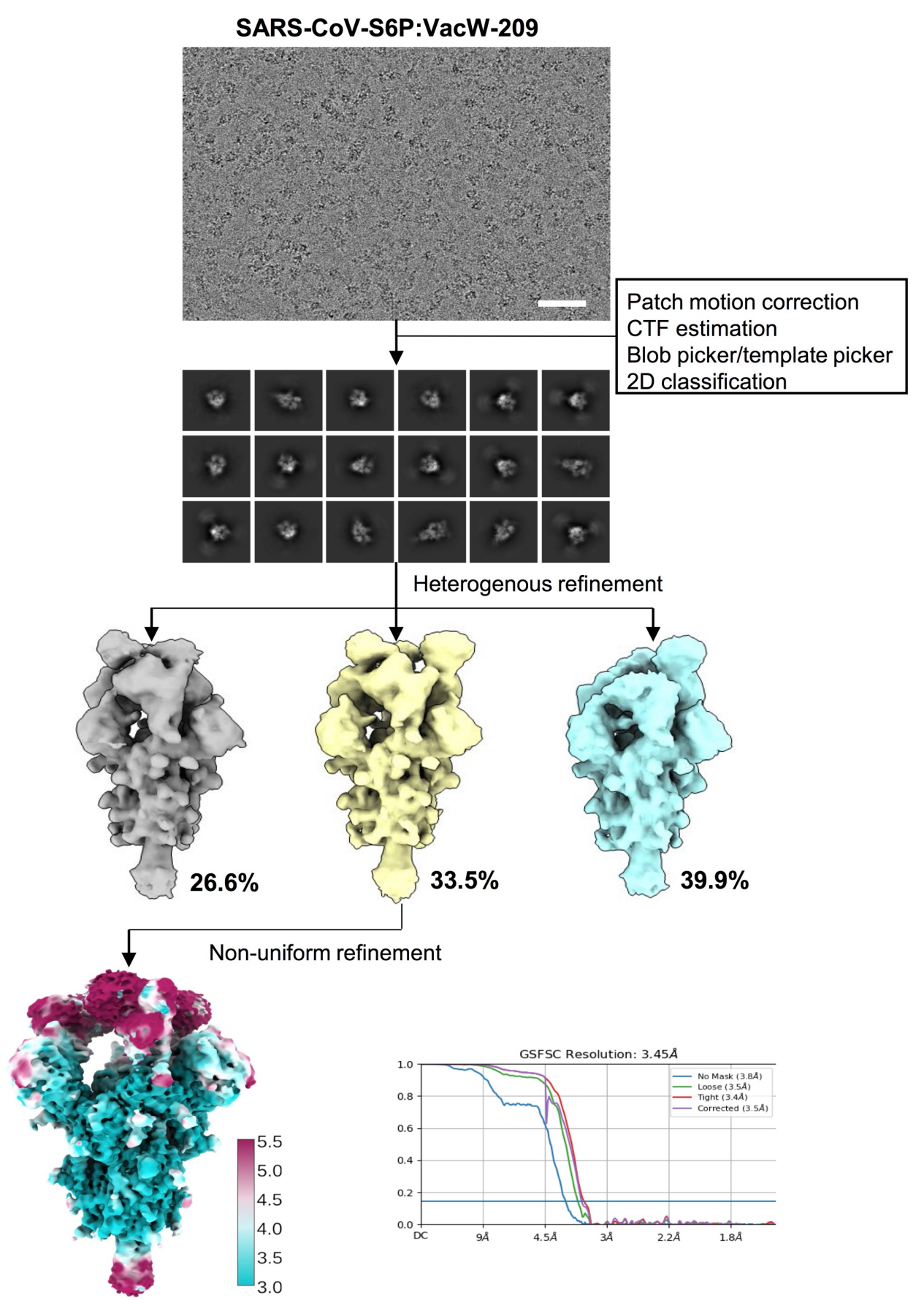

288 Figure S12. Single-particle cryo-EM images processing workflow and the 289 global and local resolution estimation for the immune complex of SARS290 CoV-S6P:VacW-209. 
A

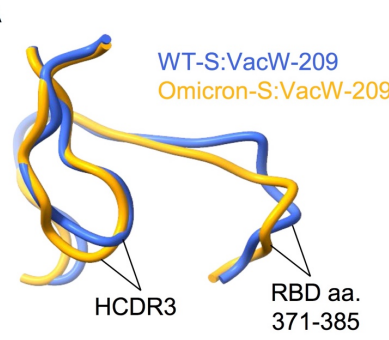

B

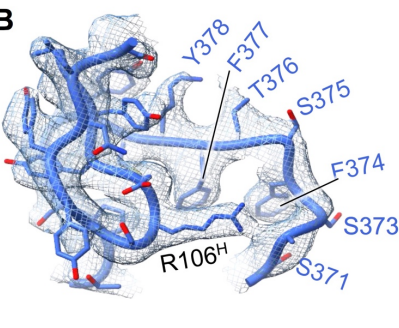

C

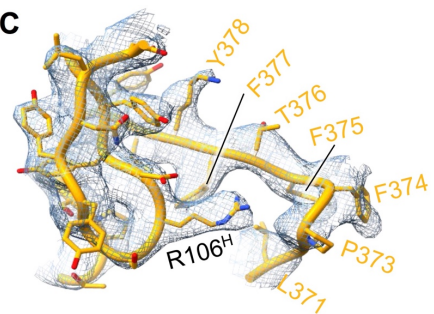

D

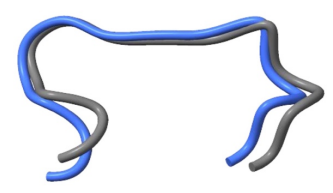

WT (VacW-209 bound) Omicron (pdb no. 7t9j)
RMSD: $1.16 \AA$
E

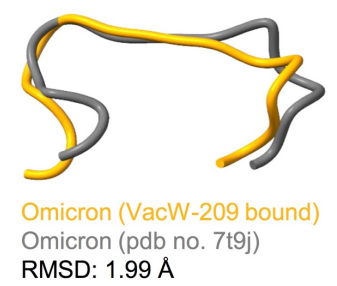

Figure S13. Structural comparison of the interactions of VacW-209 HCDR3 to WT or Omicron RBD. (A-C) The HCDR3 (aa. 104-119) and interacted RBD 294 loop (aa. 371-385) from structures of WT-S:VacW-209 (blue) and Omicron295 S:VacW-209 (orange) were superimposed shown (A) or separated shown (B296 C) with corresponding density maps. (D-E) Comparison of RBD loop (aa. 371385) from WT-S:VacW-209 (D)

(D) or Omicron-S:VacW-209

(E) to that from reported crystal structure of Omicron RBD (gray). 
A

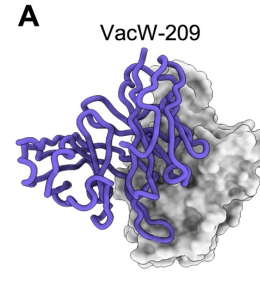

B
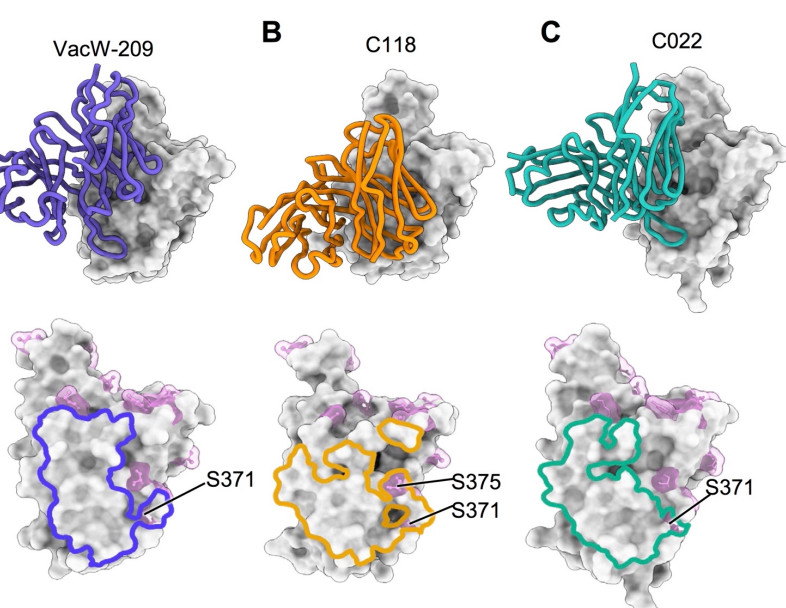

$\mathbf{F}$

\begin{tabular}{cccccc}
\hline mAbs $($ IC50) & WT & Beta & Delta & Omicron & SARS \\
\hline VacW-209 & 0.013 & 0.168 & 0.127 & 0.655 & 0.141 \\
C118 & 0.010 & 0.546 & 0.497 & 3.471 & 0.332 \\
C022 & 0.015 & 0.348 & 0.448 & 14.210 & 0.076 \\
S2X35 & 0.011 & 0.019 & 0.041 & 16.106 & 0.098 \\
S2X259 & 0.004 & 0.047 & 0.031 & 0.739 & 0.013 \\
\hline
\end{tabular}

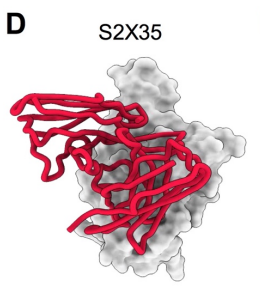

E $\quad \mathbf{S} 2 \times 259$
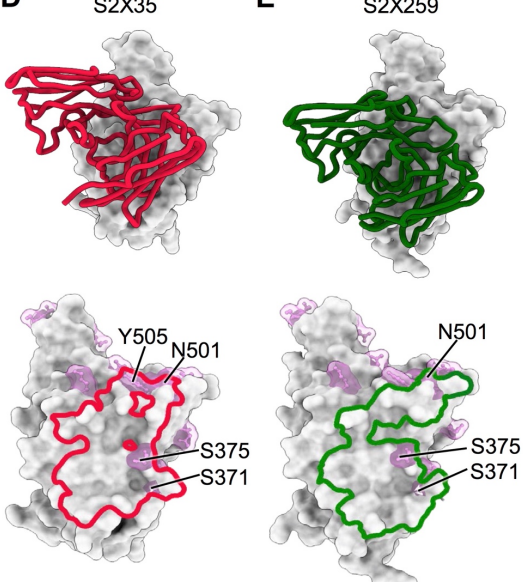

G

\begin{tabular}{ccccc}
\hline mAbs (KD) & WT & Omicron & & $\begin{array}{c}\text { Binding affinity } \\
\text { (Ko, nM) }\end{array}$ \\
\cline { 1 - 3 } VacW-209 & 0.0268 & 0.722 & \\
C118 & 0.0197 & 2.83 & 0.005 \\
C022 & 0.0356 & 2.26 & 0.1 \\
S2X35 & 0.0183 & 43.0 & & 10 \\
S2X259 & 0.00757 & 2.76 & & 50 \\
\hline
\end{tabular}

300 Figure S14. Structural comparison of VacW-209-like nAbs. (A-E) Binding 301 modes (upper) and footprints (lower) of VacW-209 (A), C118 (B) (7RKS), C022 302 (C) (7RKU), S2X35 (D) (7R6W), and S2X259 (E) (7M7W). RBDs are shown as 303 gray surface and nAbs are presented as colored cartoon. The footprints of nAbs 304 and mutations involved in nAbs interactions are labeled in the lower panels. (F) 305 The neutralization of VacW-209-like nAbs against SARS-CoV-2 WT, Beta, Delta, 306 Omicron, and SARS-CoV pseudoviruses. (G) The binding affinity of VacW-209307 like nAbs to SARS-CoV-2 WT and Omicron RBDs by SPR. The data 308 represented here was mean of at least two independent experiments. 
A

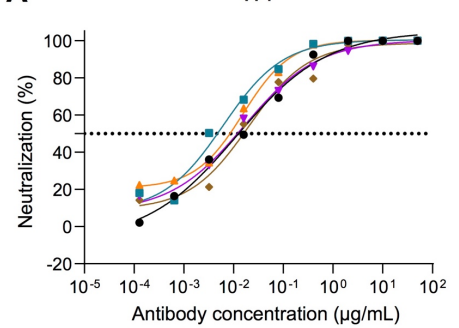

Omicron

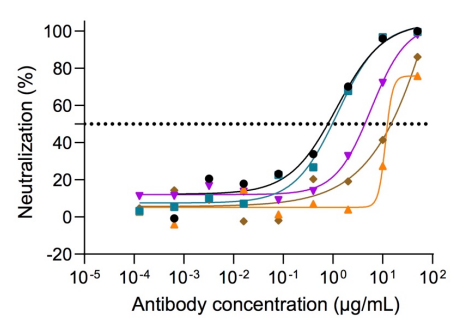

Beta

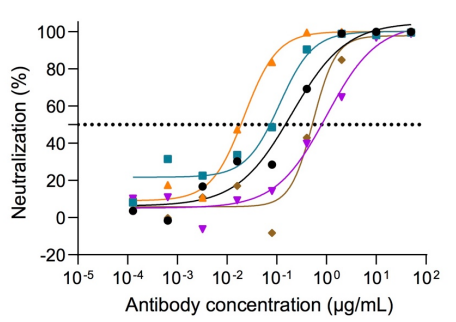

SARS-CoV

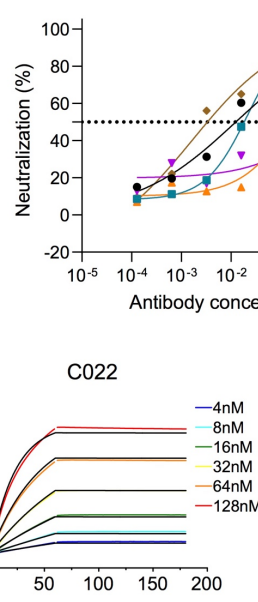

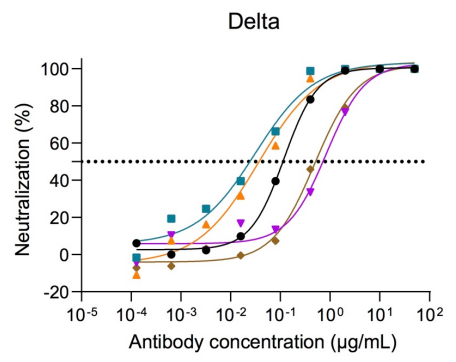

- VacW-209

$\rightarrow \mathrm{C} 118$

$-\mathrm{C} 022$

- S2X35

- S2X259

B

C118
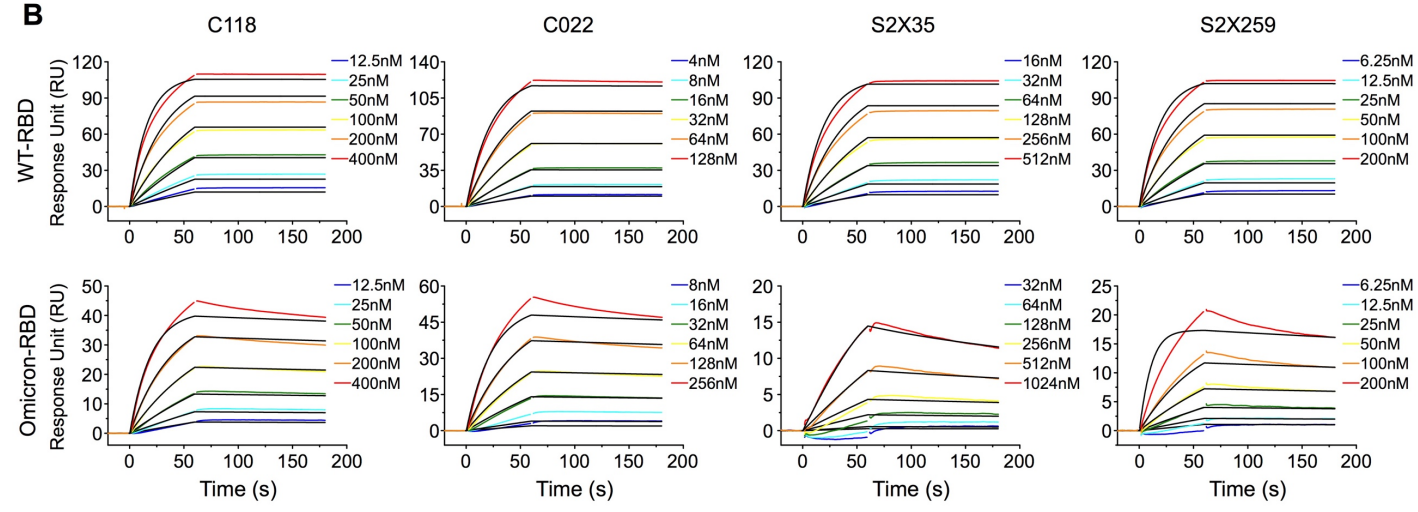

Figure S15. Neutralization (A) and binding affinity (B) curves of VacW-209-

311 like nAbs to SARS-CoV-2 WT, variants, and SARS-CoV.

312 One out of at least two independent experiments with similar results. 
Table S1. Cryo-EM data collection, refinement and validation statistics of single-antibody immune-complexes

\begin{tabular}{|c|c|c|c|c|c|c|c|c|c|c|c|c|}
\hline & $\begin{array}{l}\text { SARS-CoV-2- } \\
\text { S:209 }\end{array}$ & $\begin{array}{l}\text { SARS-CoV-2- } \\
\text { S:209- } \\
\text { interface }\end{array}$ & Delta-S:209 & $\begin{array}{l}\text { Delta-S:209- } \\
\text { interface }\end{array}$ & Mu-S:209 & $\begin{array}{l}\text { Mu-S:209- } \\
\text { interface }\end{array}$ & C.1.2-S:209 & $\begin{array}{l}\text { C.1.2-S:209- } \\
\text { interface }\end{array}$ & Omicron-S:209 & $\begin{array}{l}\text { Omicron- } \\
\text { S:209- } \\
\text { interface }\end{array}$ & & $\begin{array}{c}\text { SARS-CoV- } \\
\text { S:209 }\end{array}$ \\
\hline \multicolumn{13}{|l|}{ Data collection and processing } \\
\hline Microscope & FEI TF30 & FEI TF30 & FEI TF30 & FEI TF30 & FEI TF30 & FEI TF30 & FEI TF30 & FEI TF30 & FEI TF30 & FEI TF30 & & FEI TF30 \\
\hline Camera & K3 & K3 & K3 & K3 & K3 & K3 & K3 & K3 & K3 & K3 & & K3 \\
\hline Magnification & 39,000 & 39,000 & 39,000 & 39,000 & 39,000 & 39,000 & 39,000 & 39,000 & 39,000 & 39,000 & \# & 39,000 \\
\hline Voltage (kV) & 300 & 300 & 300 & 300 & 300 & 300 & 300 & 300 & 300 & 300 & & 300 \\
\hline Electron exposure (e-/A2) & 60 & 60 & 60 & 60 & 60 & 60 & 60 & 60 & 60 & 60 & & 60 \\
\hline Defocus range $(\mu \mathrm{m})$ & $1.2-3.5$ & $1.2-3.5$ & $1.2-3.6$ & $1.2-3.6$ & $1.0-3.0$ & $1.0-3.0$ & $1.0-3.0$ & $1.0-3.0$ & $1.0-3.2$ & $1.0-3.2$ & & $1.2-3.3$ \\
\hline Pixel size $(\AA)$ & 0.778 & 0.778 & 0.778 & 0.778 & 0.778 & 0.778 & 0.778 & 0.778 & 0.778 & 0.778 & & 0.778 \\
\hline Micrographs (total) & 5342 & 5342 & 2142 & 2142 & 8447 & 8447 & 4062 & 4062 & 8608 & 8608 & & 3700 \\
\hline Micrographs (used) & 5098 & 5098 & 2032 & 2032 & 7524 & 7524 & 3703 & 3703 & 7322 & 7322 & & 3196 \\
\hline Final particle images (no.) & 320,451 & 336,066 & 129,767 & 389,301 & 323,168 & 969,504 & 225,574 & 225,574 & 150,534 & 451,606 & & 271,929 \\
\hline Symmetry imposed & C3 & C1 & C3 & C1 & C3 & C1 & C1 & C1 & C1 & C1 & & C1 \\
\hline Map resolution $(\AA)$ & 3.27 & 3.90 & 2.98 & 3.71 & 3.09 & 3.77 & 3.15 & 3.52 & 3.39 & 3.90 & & 3.45 \\
\hline FSC threshold & 0.143 & 0.143 & 0.143 & 0.143 & 0.143 & 0.143 & 0.143 & 0.143 & 0.143 & 0.143 & & 0.143 \\
\hline Map sharpening B factor ( $(\AA 2)$ & -122.4 & -159.9 & -84.0 & -150.8 & -116.3 & -163.1 & -81.9 & -112.3 & -114.7 & -167.2 & & -127.1 \\
\hline \multicolumn{13}{|l|}{ Validation } \\
\hline MolProbity score & 1 & 1.82 & 1 & 1.86 & 1 & 1.80 & 1 & 1.87 & I & 1.80 & & 1 \\
\hline Clashscore & 1 & 5.26 & 1 & 7.10 & 1 & 4.03 & I & 6.21 & I & 5.05 & & I \\
\hline Poor rotamers (\%) & 1 & 0.28 & 1 & 0.29 & 1 & 0.00 & 1 & 0.57 & I & 0.56 & & I \\
\hline RMS (bonds) & 1 & 0.0063 & 1 & 0.0080 & 1 & 0.0060 & I & 0.0127 & 1 & 0.0055 & & 1 \\
\hline RMS (angles) & 1 & 1.23 & 1 & 0.92 & 1 & 1.30 & 1 & 1.43 & I & 1.23 & & 1 \\
\hline \multicolumn{13}{|l|}{ Ramachadran plot } \\
\hline Favored (\%) & 1 & 90.21 & 1 & 92.48 & 1 & 87.53 & I & 90,87 & 1 & 90.45 & & 1 \\
\hline Allowed (\%) & 1 & 9.79 & 1 & 7.52 & 1 & 12.23 & I & 9.13 & 1 & 9.31 & & I \\
\hline Disallowed (\%) & 1 & 0.00 & 1 & 0.00 & 1 & 0.24 & 1 & 0.00 & 1 & 0.24 & & 1 \\
\hline
\end{tabular}

